\title{
GRB spectrum from gradual dissipation in a magnetized outflow
}

\author{
Ramandeep Gill, ${ }^{1,2 \star}$ Jonathan Granot $^{1,2}$ and Paz Beniamini ${ }^{\circledR 3}$ \\ ${ }^{1}$ Department of Physics, The George Washington University, Washington, DC 20052, USA \\ ${ }^{2}$ Department of Natural Sciences, The Open University of Israel, P.O. Box 808, Ra'anana 43537, Israel \\ ${ }^{3}$ Theoretical Astrophysics, California Institute of Technology, Mail Code 350-17, Pasadena, CA 91125, USA
}

Accepted 2020 September 15. Received 2020 September 15; in original form 2020 August 24

\begin{abstract}
Modelling of many gamma-ray burst prompt emission spectra sometimes requires a (quasi) thermal spectral component in addition to the Band function that sometimes leads to a double-hump spectrum, the origin of which remains unclear. In photospheric emission models, a prominent thermal component broadened by sub-photospheric dissipation is expected to be released at the photospheric radius, $r_{\mathrm{ph}} \sim 10^{12} \mathrm{~cm}$. We consider an ultra-relativistic strongly magnetized steady outflow with a striped-wind magnetic-field structure undergoing gradual and continuous magnetic energy dissipation at $r<r_{\mathrm{s}}$ that heats and accelerates the flow to a bulk Lorentz factor $\Gamma(r)=\Gamma_{\infty} \min \left[1,\left(r / r_{\mathrm{s}}\right)^{1 / 3}\right]$, where typically $r_{\mathrm{ph}}<r_{\mathrm{s}}$. Similar dynamics and energy dissipation rates are also expected in highly variable magnetized outflows without stripes/field-reversals. Two modes of particle energy injection are considered: (a) power-law electrons, e.g. accelerated by magnetic reconnection, and (b) distributed heating of all electrons (and $\mathrm{e}^{ \pm}$-pairs), e.g. due to magnetohydrodynamic instabilities. Steady-state spectra are obtained using a numerical code that evolves coupled kinetic equations for a photon-electron-positron plasma. We find that (i) the thermal component consistently peaks at $(1+z) E_{\mathrm{pk}} \sim 0.2-1 \mathrm{MeV}$, for a source at redshift $z$, and becomes sub-dominant if the total injected energy density exceeds the thermal one, (ii) power-law electrons cool mainly by synchrotron emission whereas mildly relativistic and almost monoenergetic electrons in the distributed heating scenario cool by Comptonization on thermal peak photons, (iii) both scenarios can yield a low-energy break, and (iv) the $\sim 0.5(1+z)^{-1} \mathrm{keV} \mathrm{X}$-ray emission is suppressed in scenario (a), whereas it is expected in scenario (b). Energy-dependent linear polarization can differentiate between the two particle heating scenarios.
\end{abstract}

Key words: acceleration of particles-magnetic reconnection-MHD-radiation mechanisms: non-thermal-relativistic processes - gamma-ray burst: general.

\section{INTRODUCTION}

The emission mechanism that powers the prompt gamma-ray emission in both short-hard $\left(T_{90} \lesssim 2 \mathrm{~s}\right.$; Kouveliotou et al. 1993) and long-soft ( $T_{90} \gtrsim 2 \mathrm{~s}$ ) gamma-ray bursts (GRBs; see e.g. Piran 2004; Kumar \& Zhang 2015 for reviews) is still a matter of debate. The typical prompt emission spectrum is non-thermal and is traditionally described by the empirical 'Band function' (Band et al. 1993), representing a smoothly broken power law. The break photon energy where the $E L_{E}$ spectrum peaks is on average measured to be around $\left\langle E_{\mathrm{pk}}\right\rangle \simeq 250 \mathrm{keV}$ and the mean power-law photon indices below and above $E_{\mathrm{pk}}$ are $\langle\alpha\rangle \simeq-1$ and $\langle\beta\rangle \simeq-2.3$, respectively (e.g. Preece et al. 2000; Kaneko et al. 2006). One of the main difficulties in understanding the emission mechanism has been our ignorance of the jet composition, i.e. whether it is kinetic energy dominated (Rees \& Meszaros 1994) or Poynting-flux dominated (Thompson 1994; Lyutikov \& Blandford 2003), which dictates the mode of energy dissipation in the outflow, e.g. internal shocks or magnetic reconnection, respectively. When the outflow is loaded with protons and neutrons, nuclear collisions between the

^E-mail: rsgill.rg@gmail.com two particle species can also dissipate energy (e.g. Beloborodov 2010).

The localization of the spectral peak to $100 \mathrm{keV} \lesssim E_{\mathrm{pk}} \lesssim 1 \mathrm{MeV}$ finds a natural explanation in photospheric emission models with subphotospheric dissipation (see e.g. Beloborodov \& Mészáros 2017 for a review). In this scenario, the spectral peak and the low-energy part of the spectrum at photon energies $E<E_{\mathrm{pk}}$ are formed by quasithermal Comptonization of soft seed photons up to the thermal peak by mildly relativistic electrons when the flow is optically thick with Thomson optical depth $1 \lesssim \tau_{\mathrm{T}} \lesssim 100$ (e.g. Eichler \& Levinson 2000; Pe'er \& Waxman 2004; Rees \& Mészáros 2005; Giannios \& Spruit 2007; Beloborodov 2013; Vurm, Lyubarsky \& Piran 2013; Thompson \& Gill 2014; Bhattacharya \& Kumar 2020). Continued dissipation as the flow becomes optically thin $\left(\tau_{T}<1\right)$ then gives rise to the high-energy part of the spectrum at photon energies $E>E_{\mathrm{pk}}$ (e.g. Giannios 2006; Pe’er, Mészáros \& Rees 2006; Giannios 2008; Gill \& Thompson 2014; Vurm \& Beloborodov 2016). The end result is a broadened spectrum that resembles the typical non-thermal Band function as compared to a narrow thermal one.

Many GRBs, however, show deviations from the typical singlecomponent Band spectrum by having multiple spectral components (e.g. Guiriec et al. 2016b), namely a double-hump spectral profile (Guiriec et al. 2011, 2017) with, sometimes, an additional underlying 
power-law component that in some cases features a high-energy cutoff (Ryde 2004, 2005; Guiriec et al. 2010, 2015a,b, 2016a). When two spectral humps are present, one of them is modelled as being thermal and the other non-thermal, where the latter is generally interpreted as fast-cooling synchrotron emission from electrons with a power-law energy distribution. The emergence of the two-component spectrum in the internal-shock model (e.g. Mészáros \& Rees 2000) as well as in a magnetized outflow (e.g. Beniamini \& Giannios 2017) has been demonstrated analytically. Better and consistent time-resolved spectral fits have been obtained in the observational works mentioned above when using a two-component thermal+non-thermal model over the traditional single-component Band function. Typically, the thermal component is sub-dominant. However, in some (albeit rare) cases the entire pulse is dominated by thermal (or quasithermal) emission (e.g. Ryde 2004). The presence of the thermal component in the spectra of many bursts gave the initial motivation to consider photospheric emission models. Now, increasing incidence for such components in GRB spectra, attributed to the wider energy range of Fermi/Gamma-ray Burst Monitor as well as the use of multicomponent spectral fits, gives further credence to this idea.

In addition, many GRBs have been shown to feature a lowenergy spectral break at $E_{\mathrm{br}} \sim 0.03 E_{\mathrm{pk}}$, with photon indices $\alpha_{1}=$ $-0.66 \pm 0.35$ for $E<E_{\mathrm{br}}$ and $\alpha_{2}=-1.46 \pm 0.31$ for $E_{\mathrm{br}}<E$ $<E_{\mathrm{pk}}$ (Oganesyan et al. 2017; Ravasio et al. 2019). Such a break would be naturally produced in models that considered optically thin synchrotron emission from fast-cooling electrons, where the break would represent the cooling break due to synchrotron emission from electrons cooling at the dynamical time, with photon indices $\alpha_{1}=$ $-2 / 3$ and $\alpha_{2}=-3 / 2$ (Katz 1994; Rees \& Meszaros 1994; Tavani 1996; Sari, Piran \& Narayan 1998; Granot \& Sari 2002; Kumar \& McMahon 2008; Beniamini \& Piran 2013). Under this interpretation, the relative proximity of the two break energies suggests that the particle injection Lorentz factor (LF) is close to the cooling LF (recall that $\gamma_{m} \propto E^{1 / 2}$ ) and therefore that synchrotron emission is produced in the marginally fast cooling regime (Daigne, Bošnjak \& Dubus 2011; Beniamini, Barniol Duran \& Giannios 2018). Photospheric emission models generally lack such a break at low energies apart from that produced by synchrotron self-absorption of the soft seed photon source, which typically features a much harder RayleighJeans spectrum below $E_{\mathrm{br}}$ with $\alpha_{1}=1$.

To investigate spectral formation, detailed numerical simulations have been performed for models featuring energy dissipation in internal shocks with power-law electrons emitting synchrotron photons (Pe'er \& Waxman 2004, 2005), neutron-proton collisional heating with monoenergetic $\mathrm{e}^{ \pm}$-pair injection (Vurm, Beloborodov \& Poutanen 2011; Vurm \& Beloborodov 2016), and distributed heating with quasi-thermal Comptonization of soft photospheric component (Pe'er et al. 2006; Gill \& Thompson 2014) or self-absorbed cyclosynchrotron emission (Stern \& Poutanen 2004; Vurm et al. 2013; Thompson \& Gill 2014) as the main emission mechanisms. These works self-consistently included the effects of $\mathrm{e}^{ \pm}$-pair cascades and conducted a thorough parameter space study. Many works considered a single-collision model, in which the final spectrum was derived from dissipation occurring over a single dynamical time. Some only considered sub-photospheric dissipation in a flow coasting at its terminal bulk LF, $\Gamma_{\infty}$. Earlier numerical works that explored dissipation in a Poynting-flux dominated outflow, using Monte Carlo simulations (Giannios \& Spruit 2005; Giannios 2006; Giannios \& Spruit 2007; Giannios 2008), considered a thermal distribution of electrons at (comoving) temperature $T_{e}^{\prime}$ set by the balance between volumetric heating of all particles due to magnetic energy dissipation and their cooling due, mostly to Comptonization, as well as synchrotron emission. These works did not include the effects of $\mathrm{e}^{ \pm}$-pairs on the final spectrum.

The appearance of the double-hump spectrum and low-energy break offers additional clues for understanding the prompt GRB emission mechanism. To this end, we consider a photospheric emission model with sub-photospheric dissipation, occurring continuously from $r_{\tau 0} \ll r_{\mathrm{ph}}<r_{\mathrm{s}}$ until the saturation radius $r_{\mathrm{s}}$, from magnetic reconnection or magnetohydrodynamic (MHD) instabilities in a striped-wind Poynting-flux dominated ultra-relativistic outflow. Beniamini \& Giannios (2017) carried out analytic modelling of this scenario that yielded two-component spectra for a range of values of the model parameters. Alternatively, in a highly time-variable and magnetized relativistic outflow impulsive magnetic acceleration takes place even without any magnetic-field reversals (i.e. stripedwind) (e.g. Granot, Komissarov \& Spitkovsky 2011) that leads to a similar dynamical evolution and energy dissipation per unit radius, where dissipation occurs through internal shocks rather than magnetic reconnection (Granot et al. 2011; Granot 2012; Komissarov 2012). Therefore, the results of this work are generally applicable to a wider class of Poynting-flux-dominated models.

Here, we consider two energy dissipation scenarios that accelerate/heat the electrons (and created $e^{ \pm}$-pairs) differently. (i) Magnetic reconnection in the striped wind is assumed to accelerate a fraction $\xi$ of the total baryonic electrons in the emission region, whose initial Thomson optical depth is $\tau_{T 0}$, into a power-law energy distribution. The remaining fraction, $(1-\xi)$ of the total, forms a cold Maxwellian distribution which is initially in thermal equilibrium with the entrained thermal radiation field for $\tau_{\mathrm{T}}>\tau_{\mathrm{T} 0}$. (ii) MHD instabilities, e.g. the Kruskal-Schwarzchild instability (Lyubarsky 2010; Gill, Granot \& Lyubarsky 2018), lead to distributed heating of all the particles that form a narrowly peaked distribution at a critical energy defined by the balance between heating and cooling.

In both scenarios, energy dissipation commences at a given optical depth $\tau_{\mathrm{T} 0}$ and coupled kinetic equations for both particles and photons are self-consistently evolved using a one-zone timedependent kinetic code that includes all relevant radiation processes and interactions between both distributions. Most importantly, we include the effect of $\mathrm{e}^{ \pm}$-pair cascades that was ignored in some earlier works due to its highly non-linear nature.

The main model is presented in Section 2, where we describe the flow dynamics of an ultra-relativistic steady spherical flow (Section 2.1) followed by energy dissipation and particle acceleration (Section 2.2) and details of the thermal radiation (Section 2.3). A brief description of the one-zone code is provided in Section 3. The two particle heating scenarios are discussed in Section 4 and the results of the simulations including radial evolution of the spectrum, particle distribution, and flow parameters for scenario (i) are presented in Section 4.1. Likewise, results for the distributed heating scenario (ii) are presented in Section 4.2. In Section 5, we carry out a parameter space exploration and present spectra for different outflow parameters. Low-energy spectral breaks are discussed in Section 6 followed by a summary of this work and discussion in Section 7.

\section{GRADUAL ENERGY DISSIPATION IN A RELATIVISTIC SPHERICAL FLOW}

\subsection{Flow dynamics}

We consider a steady Poynting-flux-dominated relativistic (locally) spherical flow with a striped wind magnetic field structure (e.g. Lyubarsky \& Kirk 2001; Bégué, Pe’er \& Lyubarsky 2017), where we follow the treatment in Beniamini \& Giannios (2017) and present 
the salient points below. The characteristic length scale $(\lambda)$ over which the magnetic field lines reverse polarity is set by the size of the light cylinder $\left(r_{L}\right)$, such that $\lambda \sim \pi r_{L}=\pi c / \Omega=c P / 2=$ $1.5 \times 10^{7} P_{-3} \mathrm{~cm}$, where $\Omega=2 \pi / P$ is the central engine's rotational angular frequency, $P=10^{-3} P_{-3} \mathrm{~s}$ is the spin period, and $c$ is the speed of light. While this description of a striped wind flow is relevant for a millisecond magnetar central engine (e.g. Metzger et al. 2011), more generally a magnetized outflow from an accreting black hole arguably features stochastic flips in magnetic field polarity over length scales $\lambda \gtrsim r_{L}$ (e.g. McKinney \& Uzdensky 2012; Parfrey, Giannios \& Beloborodov 2015). It is worth pointing out that a broadly similar scenario may take place even without magnetic field flips or reversals, for a time-variable Poynting-flux-dominated outflow. In this case, impulsive magnetic acceleration leads to a very similar global flow dynamics (Granot et al. 2011) in terms of $\Gamma(r)$ and the fraction of the total energy that is dissipated up to a radius $r$, $f_{\text {dis }}(r)$. While there is no magnetic reconnection in this picture, energy dissipation is driven by internal shocks within the outflow (Granot et al. 2011; Granot 2012; Komissarov 2012) including multiple weak shocks at $r \ll r_{\mathrm{s}}$, where $\sigma \gg 1$ that gradually become more efficient and become strongest and most efficient when $\sigma \lesssim 1$ is reached at $r$ $\gtrsim r_{\mathrm{s}}$. In this scenario, the effective shell (rather than stripe) width is $\lambda \sim c t_{v}$ where $t_{v}=5 \times 10^{-4} P_{-3}$ s is the central engine's variability time, which is reflected in the observed variability time-scales of the prompt GRB emission (up to cosmological time dilation). The observed variability time is typically $\Delta t_{v}=(1+z) t_{v} \sim 1 \mathrm{~s}$, and so $\lambda \lesssim 10^{10} \mathrm{~cm}$.

Magnetic energy is dissipated in the flow when field lines of opposite polarity are brought together and undergo reconnection. The rate of reconnection is set by the inflow plasma velocity, $v_{\text {in }}=\epsilon v_{A}$, which is a fraction $\epsilon \sim 0.1$ of the Alfvén speed. For a strongly magnetized flow, the initial magnetization (ratio of magnetic to particle energy flux ratio) at the jet launching radius $r_{0}$ is

$\sigma_{0}=\frac{L_{B, \Omega, 0}}{L_{k, \Omega, 0}}=\frac{\beta_{0} c\left(B_{0} r_{0}\right)^{2}}{4 \pi \Gamma_{0} \dot{M}_{\Omega} c^{2}}=\frac{B_{0}^{\prime 2}}{4 \pi n_{0}^{\prime} m_{p} c^{2}} \gg 1$.

Here, $\quad L_{B, \Omega, 0}=\beta_{0} c\left(\Gamma_{0} B_{0}^{\prime} r_{0}\right)^{2} / 4 \pi \quad$ and $\quad L_{k, \Omega, 0}=\Gamma_{0} \dot{M}_{\Omega} c^{2}=$ $\beta_{0}\left(r_{0} \Gamma_{0}\right)^{2} n_{0}^{\prime} m_{p} c^{3}$ are the initial power per unit solid angle carried by the magnetic field, with comoving strength $B_{0}^{\prime}=B_{0} / \Gamma_{0}$, and kinetic power per unit solid angle carried by the cold baryons, with comoving number density $n_{0}^{\prime}$. The flow is assumed to achieve magnetization $\sigma_{\mathrm{A}}=\sigma_{0}^{2 / 3}$ at the Alfvén radius $r_{\mathrm{A}} \sim$ few $\times r_{L}$ (Drenkhahn 2002), at which point its proper velocity is $u_{A}=\left(\Gamma_{A}^{2}-1\right)^{1 / 2}=\sqrt{\sigma_{A}}$ and $\beta_{A}=u_{\mathrm{A}} / \Gamma_{\mathrm{A}}=\left(1-\Gamma_{\mathrm{A}}^{-2}\right)^{1 / 2}=v_{\mathrm{A}} / c=\sigma_{\mathrm{A}} /\left(1+\sigma_{\mathrm{A}}\right) \approx 1$, and therefore $v_{\text {in }}=\epsilon c$.

Under the assumption that a reasonable fraction of the dissipated energy in the flow goes towards its acceleration, the condition $\Gamma(r) \sigma(r)=\Gamma_{0} \sigma_{0}$ always holds a long as $\sigma \gg 1$ [more generally $\Gamma(r)(1+\sigma(r))=\Gamma_{0}\left(1+\sigma_{0}\right]$ from conservation of the total specific energy, i.e. neglecting radiative losses etc., where $\sigma=B^{2} / 4 \pi w$ and $w$ is the proper enthalpy density, which eventually leads to $\Gamma\left(r>r_{\mathrm{s}}\right) \approx \Gamma_{\infty} \approx \Gamma_{0} \sigma_{0}=\sigma_{0}=\sigma_{\mathrm{A}}^{3 / 2}$, where $\sigma\left(r>r_{\mathrm{s}}\right)<1$ (see e.g. Granot et al. 2011). At this point, the flow becomes kinetic energy dominated and starts to coast at its terminal LF $\Gamma_{\infty}$ until it is decelerated by its interaction with the external medium - interstellar medium (ISM) for short-hard GRBs and stellar wind of the massive star progenitor of long-soft GRBs. Beyond the Alfvén radius the outflow's bulk LF grows as a power law in radius

$\Gamma(r)=\Gamma_{\infty}\left(\frac{r}{r_{\mathrm{s}}}\right)^{1 / 3}, \quad r_{\mathrm{A}}<r<r_{\mathrm{s}}$, until the saturation radius, ${ }^{1}$

$r_{\mathrm{s}}=\frac{\Gamma_{\infty}^{2} \lambda}{6 \epsilon}=1.7 \times 10^{13} \Gamma_{\infty, 3}^{2}\left(\frac{\lambda}{\epsilon}\right)_{8} \mathrm{~cm}$,

at which point all of the magnetic energy in the flow has been dissipated with nothing left for further acceleration. However, further dissipation can still occur due to internal shocks that become efficient when $\sigma<1$ for $r>r_{\mathrm{s}}$, as argued above.

The flow is launched Poynting-flux dominated and the total power per unit solid angle crossing radius $r$ is given by $L_{\Omega}=$ $L_{B, \Omega}+L_{k, \Omega}+L_{\gamma, \Omega}$, where the last term represents the emitted radiation. In the absence of any dissipation $L_{\gamma, \Omega}=0$, and the power carried by the Poynting flux can be expressed in terms of the total jet power, $L_{B, \Omega}=L_{\Omega}\left(1-\Gamma / \Gamma_{\infty}\right) \approx L_{\Omega}$ for $r_{\mathrm{A}}<r \ll r_{\mathrm{s}}$ (where 1 $<\Gamma \ll \Gamma_{\infty}$ and $\beta \approx 1$ ), which yields an estimate of the comoving magnetic field:

$B^{\prime} \approx\left(\frac{4 \pi L_{\Omega}}{\Gamma^{2} r^{2} c}\right)^{1 / 2}=4.1 \times 10^{6} \frac{L_{\Omega, 52}^{1 / 2}\left(\frac{\lambda}{\epsilon}\right)_{8}^{1 / 3}}{r_{12}^{4 / 3} \Gamma_{\infty, 3}^{1 / 3}} \mathrm{G}$.

The comoving number density of the baryonic electrons in the flow is given by $n^{\prime}=L_{\Omega} / r^{2} \Gamma \Gamma_{\infty} m_{p} c^{3} \propto\left(r^{2} \Gamma\right)^{-1}$, which contributes a characteristic Thomson optical depth of

$\tau_{T}=\frac{n^{\prime} \sigma_{T} r}{\Gamma}=\frac{\sigma_{T} L_{\Omega}}{r \Gamma^{2} \Gamma_{\infty} m_{p} c^{3}}=1 \frac{L_{\Omega, 52}\left(\frac{\lambda}{\epsilon}\right)_{8}^{2 / 3}}{\Gamma_{\infty, 3}^{5 / 3} r_{12}^{5 / 3}}$,

where $\sigma_{\mathrm{T}}$ is the Thomson cross-section. For $r<r_{\mathrm{s}}, \Gamma \propto r^{1 / 3}$, and therefore $\tau_{T} \propto r^{-5 / 3}$. However, when the flow starts to coast at $\Gamma=\Gamma_{\infty}$ the Thomson optical depth drops more slowly with radius, $\tau_{T} \propto r^{-1}$. At $\tau_{T}=1$ matter and radiation decouple, allowing the radiation to stream freely, which defines the photospheric radius,

$r_{\mathrm{ph}} \approx 10^{12} \frac{L_{\Omega, 52}^{3 / 5}\left(\frac{\lambda}{\epsilon}\right)_{8}^{2 / 5}}{\Gamma_{\infty, 3}} \mathrm{~cm}$.

Here, we have only considered the Thomson optical depth of baryonic electrons. In Fig. 1, we show the different parameters for which $r_{\mathrm{ph}}=$ $r_{\mathrm{s}}$. The vertical axis shows the typical range expected for $\left(\frac{\lambda}{\epsilon}\right)_{8}$, where the lower end is relevant for a millisecond magnetar central engine and the higher end reflects the typical values based on the observed variability time-scale of prompt GRB emission. The solid lines show the model parameter space for fixed values of the jet power per unit solid angle, $L_{\Omega} \lesssim 10^{52} \mathrm{erg} \mathrm{s}^{-1} \mathrm{sr}^{-1}$, the fiducial value adopted in this work. We consider the regime with $r_{\mathrm{ph}}<r_{\mathrm{s}}$ when the flow is heated continuously, as it transitions from the optically thick to thin regimes. We will show below that copious pair-production ensues when energy dissipation leads to particle acceleration into a powerlaw energy distribution that emits energetic synchrotron radiation. The created pairs extend the photospheric radius by factors of a few.

\subsection{Energy dissipation and particle acceleration}

Energy is dissipated gradually in the flow, for $r<r_{\mathrm{s}}$, as magnetic field lines of opposite polarity come into contact and undergo magnetic reconnection. The rate of energy dissipation at any given radius can be obtained from the Poynting-flux power, such that (Giannios \&

\footnotetext{
${ }^{1}$ Throughout this work, the notation $Q_{x}$ denotes the value of the quantity $Q$
} in units of $10^{x}$ times its (cgs) units 


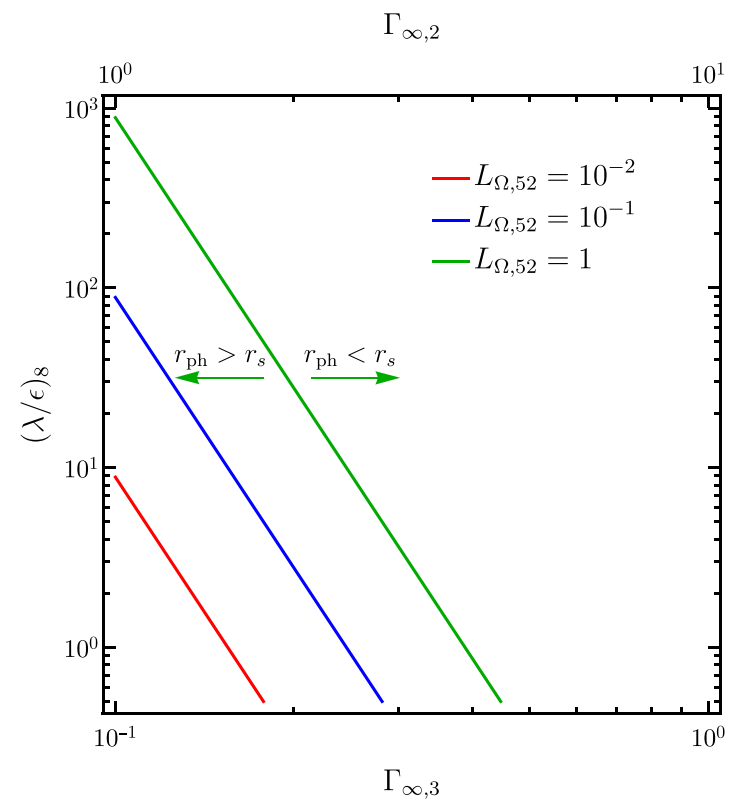

Figure 1. Parameter space for which the photospheric radius $r_{\mathrm{ph}}$, due to baryonic electrons, is equal to the saturation radius $r_{s}$, shown as a function of $\Gamma_{\infty}$ and $(\lambda / \epsilon)$ for fixed jet power per unit solid angle $L_{\Omega}$ (note that the outflow's total isotropic equivalent power is $\left.4 \pi L_{\Omega}=1.26 \times 10^{53} L_{\Omega, 52} \mathrm{erg} \mathrm{s}^{-1}\right)$. We only consider the regime where $r_{\mathrm{ph}}<r_{\mathrm{S}}$ (to the right of the lines) when the flow is heated continuously from the optically thick to thin regime.

Spruit 2005)

$$
\begin{aligned}
\frac{\mathrm{d} L_{\mathrm{diss}, \Omega}}{\mathrm{d} r} & =-\frac{\mathrm{d} L_{B, \Omega}}{\mathrm{d} r}=-\frac{\mathrm{d}}{\mathrm{d} r}\left[L_{\Omega}\left(1-\frac{\Gamma}{\Gamma_{\infty}}\right)\right] \\
& =\frac{1}{3} \frac{L_{\Omega}}{\Gamma_{\infty}} \frac{\Gamma}{r} \propto r^{-2 / 3} .
\end{aligned}
$$

This implies a differential dissipation $\mathrm{d} L_{\text {diss, } \Omega} \propto r^{-2 / 3} \mathrm{~d} r$ or a cumulative dissipation $L_{\text {diss, } \Omega}(<r) \propto r^{1 / 3}$ at $r_{0}<r<r_{\mathrm{s}}$. At $r=r_{\mathrm{s}}$, when $\Gamma=\Gamma_{\infty}$, magnetic energy dissipation peaks and stops, so that $f_{\text {dis }}(r)=L_{\text {diss }, \Omega}(<r) / L_{\Omega}=\min \left[1,\left(r / r_{s}\right)^{1 / 3}\right]$. Next, we relate the dissipated power to the comoving dissipated energy density, $\mathrm{d} L_{\mathrm{diss}, \Omega}=r^{2} \Gamma^{2} c \mathrm{~d} U_{\mathrm{diss}}^{\prime}$, and express $\mathrm{d} r=\Gamma \beta c \mathrm{~d} t^{\prime} \approx \Gamma c \mathrm{~d} t^{\prime}$ for $\Gamma$ $\gg 1$ and $\beta \approx 1$, which yields

$\frac{\mathrm{d} U_{\mathrm{diss}}^{\prime}}{\mathrm{d} t^{\prime}}=\frac{1}{3} \frac{L_{\Omega}}{\Gamma_{\infty} r^{3}}$.

Magnetic reconnection leads to the acceleration of electrons into a non-thermal power-law energy distribution, with $\mathrm{d} n^{\prime} \propto \gamma_{\mathrm{e}}^{-p} \mathrm{~d} \gamma_{\mathrm{e}}$ for $\gamma_{m}<\gamma_{\mathrm{e}}<\gamma_{M}$, for which the mean energy per unit rest mass is $\left\langle\gamma_{\mathrm{e}}\right\rangle_{\mathrm{nth}}=[(p-1) /(p-2)] \gamma_{m}$ when $p>2$. The power-law index $p$ has been shown to depend sensitively on the value of $\sigma$ (e.g. Sironi \& Spitkovsky 2014; Guo et al. 2015; Kagan et al. 2015; Werner et al. 2016), where it can be approximated to follow the scaling (Beniamini \& Giannios 2017)

$p=4 \sigma^{-0.3}$.

In models featuring internal shocks, $2 \lesssim p \lesssim 3$ is left to vary as one of the model parameters, whereas the $\sigma$ dependence of $p$, as employed here, reduces the total number of model parameter by one.

It is assumed here for simplicity that half of the dissipated energy $E_{\text {diss }}^{\prime}$ goes directly into the flow's kinetic energy (see e.g. Drenkhahn \& Spruit 2002), while the other half goes towards particle acceleration and is divided between electrons $\left(\epsilon_{e} E_{\text {diss }}^{\prime} / 2\right)$ and protons $\left(\left(1-\epsilon_{e}\right) E_{\text {diss }}^{\prime} / 2\right)$, where most of the latter energy is also typically quickly converted into kinetic energy. In scenario $(i)$, we further assume that only a fraction $\xi<1$ of electrons are actually accelerated during magnetic reconnection, and the remaining fraction $(1-\xi)$ form a thermal distribution. The mean energy per baryon is limited to $\sigma m_{p} c^{2}$, as this is the total dissipated energy per baryon-electron for complete magnetic dissipation; however, some particle may in principle exceed the mean energy. Therefore, the mean energy per accelerated electron, for a total of $N_{\mathrm{e}}$ electrons, is given as $\xi\left\langle\gamma_{e}\right\rangle m_{e} c^{2}=\left(\epsilon_{e} / 2\right) E_{\text {diss }}^{\prime} / N_{e}=\epsilon_{e} \sigma m_{p} c^{2} / 2$, which yields an estimate of the mean energy per rest mass energy of the non-thermal electrons (Beniamini \& Giannios 2017)

$$
\left\langle\gamma_{e}\right\rangle_{\mathrm{nth}}=\frac{\epsilon_{e}}{2 \xi} \sigma \frac{m_{p}}{m_{e}}=2.3 \times 10^{3}\left(\frac{\epsilon_{e}}{\xi}\right) \frac{\left(\frac{\lambda}{\epsilon}\right)_{8}^{1 / 3} \Gamma_{\infty, 3}^{2 / 3}}{r_{12}^{1 / 3}} .
$$

For a given set of flow parameters, the ratio of the parameters $\epsilon_{e}$ and $\xi$ controls the mean energy of the power-law accelerated electrons. Since $\epsilon_{\mathrm{e}}$ also controls the amount of energy put into the power-law electrons, it also sets the normalization of the non-thermal synchrotron emission component with respect to thermal component.

\subsection{Thermal radiation}

The magnetic energy in the flow is dissipated over a range of radii $\left(r_{0}<r<r_{\mathrm{s}}\right)$ and as the flow expands to larger radii its Thomson optical depth drops. Therefore, for a given set of model parameters, it is possible that energy dissipation proceeds continuously from the optically thick to thin regions. Where most of the energy is dissipated has consequences for the emergent radiation field spectrum. If most of the dissipation occurs at smaller radii, when the flow is optically thick $\left(\tau_{T} \gg 1\right)$, Compton interactions between the electrons (or pairs) and the radiation field ensure that the flow maintains (quasi)thermal equilibrium. In this case, the flow expands adiabatically and since it is radiation-dominated, the scaling of comoving energy density with comoving volume follows $U_{\text {th }}^{\prime} \propto V^{\prime-4 / 3}$. The energy density of the thermal radiation field can be related to its comoving temperature, $U_{\mathrm{th}}^{\prime}=\left(4 \sigma_{\mathrm{SB}} / c\right) T_{\mathrm{th}}^{\prime 4}$, where $\sigma_{\mathrm{SB}}$ is the Stefan-Boltzmann constant, which yields $T_{\mathrm{th}}^{\prime} \propto V^{\prime-1 / 3}$. For a steady relativistic spherical flow expanding radially, the continuity equation yields, $r^{2} \Gamma(r) n^{\prime} v=$ constant, so that $V \propto r^{2} \Gamma(r)$. This finally implies that $T_{\mathrm{th}}^{\prime}(r) \propto r^{-7 / 9}$ when $\Gamma \propto r^{1 / 3}$. The scaling of the thermal luminosity with radius can now be expressed as $L_{\mathrm{th}, \Omega}=(4 / 3) r^{2} \Gamma^{2} c U_{\mathrm{th}}^{\prime} \propto r^{-4 / 9}$. If an amount $\mathrm{d} L_{\text {diss, } \Omega}$ of power is dissipated at radius $r_{\text {diss }}$, then the thermal luminosity surviving till any radius $r>r_{\text {diss }}$ is given by $\mathrm{d} L_{\text {th, } \Omega}(r)=(1 / 2) \mathrm{d} L_{\text {diss, } \Omega}\left(r_{\text {diss }}\right)\left(r / r_{\text {diss }}\right)^{-4 / 9}$, such that the integrated thermal luminosity is, $\int_{0}^{r} \mathrm{~d} L_{\mathrm{th}, \Omega}(r) \propto r^{1 / 3}$, for $r<r_{\mathrm{s}}$, and its value at the photosphere is

$$
\begin{aligned}
L_{\mathrm{th}, \Omega}\left(r_{\mathrm{ph}}\right) & \approx \frac{3}{14} L_{\Omega}\left(\frac{r_{\mathrm{ph}}}{r_{s}}\right)^{1 / 3} \\
& =8.3 \times 10^{50} \frac{L_{\Omega, 52}^{6 / 5}}{\Gamma_{\infty, 3}\left(\frac{\lambda}{\epsilon}\right)_{8}^{1 / 5}} \operatorname{erg~s}^{-1} \mathrm{sr}^{-1},
\end{aligned}
$$

and its comoving temperature at the photosphere is

$k_{B} T_{\mathrm{th}}^{\prime}\left(r_{\mathrm{ph}}\right)=k_{B}\left(\frac{3 L_{\mathrm{th}, \mathrm{ph}, \Omega}}{16 r_{\mathrm{ph}}^{2} \Gamma_{\mathrm{ph}}^{2} \sigma_{\mathrm{SB}}}\right)^{1 / 4} \simeq 0.2 \frac{\Gamma_{\infty, 3}^{1 / 4}}{L_{\Omega, 52}^{1 / 10}\left(\frac{\lambda}{\epsilon}\right)_{8}^{3 / 20}} \mathrm{keV}$,

and the corresponding observed energy of the Wien peak is

$$
E_{\mathrm{pk}, \mathrm{th}}\left(r_{\mathrm{ph}}\right)=\frac{\Gamma_{\mathrm{ph}}}{1+z} 3 k_{B} T_{\mathrm{th}}^{\prime}\left(r_{\mathrm{ph}}\right)=\frac{210}{1+z} \frac{L_{\Omega, 52}^{1 / 10} \Gamma_{\infty, 3}^{1 / 4}}{\left(\frac{\lambda}{\epsilon}\right)_{8}^{7 / 20}} \mathrm{keV},
$$


where $k_{B}$ is the Boltzmann constant. The above peak energy estimate corresponds to that for the spectral luminosity $L_{E}$. The $E L_{E}$ Wien spectrum peak energy occurs at $4 \Gamma k_{B} T_{\mathrm{th}}^{\prime} /(1+z)$ instead. Since $T_{\mathrm{th}}^{\prime}(r) \propto r^{-7 / 12}$, the peak energy scaling with radius is $E_{\mathrm{th}, \mathrm{pk}} \propto$ $r^{-1 / 4} \propto \tau_{T 0}^{3 / 20}$. In deriving the estimates above, we have made the assumption that deeper in the flow, at very large optical depths, the energy imparted to particles is readily thermalized and the efficiency of thermalization is high.

Several works have studied the importance of the various radiative processes that shape the (quasi-)thermal spectrum at different optical depths (e.g. Beloborodov 2013; Vurm et al. 2013; Thompson \& Gill 2014; Bégué \& Pe'er 2015; Vurm \& Beloborodov 2016) and its radiative efficiency in a Poynting flux dominated flow (Pe'er 2017). The radiation field is able to maintain a blackbody spectrum only at extremely high optical depths $\left(\tau_{T} \gg 10^{2}\right)$, where softer seed photons are provided by double Compton scattering and/or bremsstrahlung (in a weakly magnetized flow, $\sigma \ll 1$ ) or cyclo-synchrotron emission (in a strongly magnetized flow, $\sigma>1$ ). At larger radii, the efficiency of completely thermalizing the flow drops and a Wien spectrum emerges instead at $\tau_{T} \gtrsim 10^{2}$. Further dissipation at lower optical depths, but still below the photosphere, acts to broaden the Wien spectrum, producing a softer spectral slope below the spectral peak energy and a harder one above it.

\section{NUMERICAL TREATMENT}

We model the emission region using a one-zone kinetic code (see Gill \& Thompson 2014, for code details), where we include all relevant high-energy radiation processes in a relativistic photon$e^{ \pm}$-pair plasma, including Compton scattering, cyclo-synchrotron emission and self-absorption, pair production and annihilation, and Coulomb interactions among the pairs.

The escape of radiation from an optically thin $\left(\tau_{T}<1\right)$ region of comoving causal size $r / \Gamma(r)$ is implemented using a simple 'leakybox' geometrical prescription (see e.g. Lightman \& Zdziarski 1987). When the flow is optically thick $\left(\tau_{T}>1\right)$, radiation is assumed to remain within the dissipation region with no leakage. To obtain the steady-state spectrum in the observer frame, we integrate over the comoving spectral emissivity (see e.g. Granot, Piran \& Sari 1999) from the photospheric radius, $r_{\mathrm{ph}}(\tilde{\theta})$, which depends on the polar angle $\tilde{\theta}$ measured from the line-of-sight (Abramowicz, Novikov \& Paczynski 1991; Pe'er 2008; Beloborodov 2011), to a large radius $\gg \max \left(r_{\mathrm{s}}, r_{\mathrm{ph}}\right)$ where $\tau_{T} \ll 1$ and the emission and absorption become negligible.

Since we employ a one-zone code, which lacks any spatial and angular information of the flow and the radiation field, the emission is approximated to arise from essentially a blob of comoving causal size $r / \Gamma$ that is radially localized at $r$ and moving with bulk LF $\Gamma(r)$. In addition, the leaky-box prescription is not particularly well suited to describe the optically thin parts of the flow when radiation is expected to stream freely. Instead, under the current prescription radiation leaks out over a (comoving) dynamical time, $t_{\mathrm{dyn}}^{\prime}=r / \Gamma c$, at the rate of $\mathrm{d} n_{\gamma}^{\prime} / \mathrm{d} t^{\prime}=-n_{\gamma}^{\prime} / t_{\text {dyn }}^{\prime}$ where $n_{\gamma}^{\prime}$ is the comoving number density of photons. Then, for a coasting flow, for which $t^{\prime} \propto r$, this would mean that the remaining photon number density, $n_{\gamma}^{\prime}(r)=n_{\gamma, 0}^{\prime}\left(r_{0} / r\right)$, is still half at $r=r_{0}+\Delta r=2 r_{0}$ of that emitted a dynamical time (radius doubling time) ago at $r=r_{0}$. As a result, the radiation field accumulates in the emission region over multiple dynamical times, which is unphysical and may produce some artefacts. For example, this would cause a larger suppression of the high-energy part of the spectrum due to $\gamma \gamma$-annihilation for which a test photon with energy $E>\Gamma m_{\mathrm{e}} c^{2} /(1+z)$ 'sees' a larger optical depth $\tau_{\gamma \gamma}$ due to larger number density of annihilating low-energy target photons at energy $\sim\left(\Gamma m_{e} c^{2}\right)^{2} / E(1+z)^{2}$. This also leads to the emergence of a power-law spectral break at high-energies instead of an exponential one (e.g. Granot, Cohen-Tanugi \& Silva 2008). Therefore, a more accurate radiation transfer treatment, which is outside the scope of this work, is needed to avoid such artefacts and include the angular dependence of the radiation field (see e.g. Vurm \& Beloborodov 2016).

\section{TWO DIFFERENT PARTICLE HEATING SCENARIOS}

Magnetic energy dissipation due to either magnetic reconnection or MHD instabilities commences when the flow is highly optically thick. It continues to inject energy in the form of either power-law (baryonic) electrons or via distributed heating of all particles, respectively. The details of how particle injection/heating is implemented in the simulation are presented in the Appendix.

Our starting point is an optically thick flow with initial Thomson optical depth $\tau_{\mathrm{T} 0}=100$. At this point, the comoving radiation field spectrum resembles a Wien-like thermal spectrum,

$$
\frac{\mathrm{d} n_{\gamma}^{\prime}}{\mathrm{d} \ln E^{\prime}}=\frac{U_{0}^{\prime}}{6\left(k_{B} T_{\mathrm{th}}\right)^{4}} E^{\prime 3} \exp \left(-\frac{E^{\prime}}{k_{B} T_{\mathrm{th}}^{\prime}}\right)
$$

characterized by its temperature $T_{\text {th }}^{\prime}$ from equation (12) and normalization given by $U_{0}^{\prime}=L_{\mathrm{th}, \Omega} /(4 / 3) r^{2} \Gamma^{2} c$ with $L_{\mathrm{th}, \Omega}=$ $L_{\mathrm{th}, \mathrm{ph}, \Omega}\left(r / r_{\mathrm{ph}}\right)^{1 / 3}=L_{\mathrm{th}, \mathrm{ph}, \Omega} \tau_{T}^{-1 / 5}$ in equation (11) for $r<r_{\mathrm{s}}$.

\subsection{Injection of power-law electrons}

Power-law electrons injected with $\gamma_{\mathrm{e}}>\gamma_{m}=[(p-2) /(p-$ 1)] $\left\langle\gamma_{\mathrm{e}}\right\rangle_{\mathrm{nth}}$, where the last equality is valid for $p>2$ which is obtained for $\sigma<10$, emit synchrotron radiation for which the peak of the $v F_{v}$ or $E L_{E}$ synchrotron spectrum occurs at the characteristic energy (with $p=4$ when $\sigma=1$ at $r=r_{\mathrm{s}}$ according to our parametrization in equation 9)

$$
\begin{aligned}
E_{m} & =\frac{\Gamma}{1+z} h v_{m}^{\prime}=\frac{\Gamma}{1+z} \gamma_{m}^{2}\left(\frac{\hbar e B^{\prime}}{m_{\mathrm{e}} c}\right) \\
& \approx \frac{1.3 \times 10^{5}}{1+z}\left(\frac{p-2}{p-1}\right)^{2}\left(\frac{\epsilon_{\mathrm{e}}}{\xi}\right)^{2} \frac{L_{\Omega, 52}^{1 / 2} \Gamma_{\infty, 3}^{4 / 3}\left(\frac{\lambda}{\epsilon}\right)_{8}^{2 / 3}}{r_{12}^{5 / 3}} \mathrm{keV} \\
& \approx \frac{530}{1+z}\left(\frac{\epsilon_{\mathrm{e}}}{\xi}\right)^{2} \frac{L_{\Omega, 52}^{1 / 2}}{\Gamma_{\infty, 3}^{2}\left(\frac{\lambda}{\epsilon}\right)_{8}} \mathrm{keV} \quad(p=4)
\end{aligned}
$$

when particles are fast-cooling, i.e. when the characteristic cooling break energy $E_{\mathrm{c}}<E_{m}$, where

$$
E_{c}=\frac{36 \pi^{2}}{1+z} \frac{\hbar e m_{\mathrm{e}} c^{3}}{\sigma_{\mathrm{T}}^{2}} \frac{\Gamma^{3}}{B^{\prime 3} r^{2}} \approx \frac{2.6 \times 10^{-9}}{1+z} \frac{\Gamma_{\infty, 3}^{2} r_{12}^{3}}{L_{\Omega, 52}^{3 / 2}\left(\frac{\lambda}{\epsilon}\right)_{8}^{2}} \mathrm{keV},
$$

and $h=2 \pi \hbar$ is the Planck's constant. Another characteristic break in the synchrotron spectrum appears when the emission becomes self-absorbed by the emitting electrons. A simple estimate of the self-absorption break energy can be obtained by noticing that at $E=E_{\text {sa }}$ the synchrotron specific intensity cannot exceed that of a blackbody. We approximate the latter using the RayleighJeans specific intensity, $I_{E^{\prime}}^{\prime \mathrm{RJ}}\left(E_{\mathrm{sa}}^{\prime}\right)=\left(2 E_{\mathrm{sa}}^{\prime 2} / h^{3} c^{2}\right) \gamma_{\mathrm{e}}\left(E_{\mathrm{sa}}^{\prime}\right) m_{\mathrm{e}} c^{2}$, where $\gamma_{e}\left(E_{\mathrm{sa}}^{\prime}\right)=\left(E_{\mathrm{sa}}^{\prime} m_{e} c / e \hbar B^{\prime}\right)^{1 / 2}$ is the LF of electrons radiating at the self-absorption energy. The synchrotron specific intensity can be obtained from $I_{E^{\prime}}^{\prime} \sim\left(P_{E^{\prime}}^{\prime} / 4 \pi\right) \xi n^{\prime}(R / \Gamma)$, where $R / \Gamma$ is the comoving 
size of the emission region and $\xi n^{\prime}$ is the number density of baryonic electrons that were accelerated into a power-law distribution. The synchrotron spectral power at $E^{\prime}=E_{\mathrm{sa}}^{\prime}$ is given by $P_{E^{\prime}}^{\prime}\left(E_{\mathrm{sa}}^{\prime}\right)=P_{E^{\prime} \text {,max }}^{\prime}\left(E_{\mathrm{sa}}^{\prime} / E_{c}^{\prime}\right)^{-1 / 2}$ for $E_{c}^{\prime}<E_{\mathrm{sa}}^{\prime}<E_{m}^{\prime}$. The peak spectral power at $E^{\prime}=E_{c}^{\prime}$ can be approximated using the total power emitted by a single electron, $P_{\text {syn }}^{\prime}=(4 / 3) \sigma_{T} c \gamma_{e}^{2}\left(B^{\prime 2} / 8 \pi\right)$, at the characteristic synchrotron energy, $E_{\mathrm{syn}}^{\prime}=\gamma_{e}^{2} e \hbar B^{\prime} / m_{e} c$, such that $P_{E^{\prime} \text {, } \max }^{\prime} \sim P_{\mathrm{syn}}^{\prime} / E_{\mathrm{syn}}^{\prime}=\sigma_{T} B^{\prime} m_{e} c^{2} / 3 e h$. From $I_{E^{\prime}}^{\prime R J}=I_{E^{\prime}}^{\prime}$, we find the synchrotron self-absorption energy

$$
\begin{aligned}
E_{\mathrm{sa}} & \sim \frac{\Gamma}{1+z}\left(\frac{h^{3}}{8 \pi m_{p}} \frac{\xi L_{\Omega}}{\Gamma_{\infty}} \frac{1}{r^{2} \Gamma}\right)^{1 / 3} \\
& \approx \frac{1.4}{1+z} \frac{\xi^{1 / 3} L_{\Omega, 52}^{1 / 3}}{\Gamma_{\infty, 3}^{1 / 9}\left(\frac{\lambda}{\epsilon}\right)_{8}^{2 / 9} r_{12}^{4 / 9}} \mathrm{keV} .
\end{aligned}
$$

This estimate is only valid when $E_{c}<E_{\mathrm{sa}}<E_{m}$. In addition, it only accounts for the number of baryonic electrons and not the total number of particles that includes the $e^{ \pm}$-pairs, and therefore, the true value is slightly higher by a factor $\left(\tau_{\mathrm{T}} / \tau_{\mathrm{T}, \mathrm{e}}\right)^{1 / 3}$, where $\tau_{\mathrm{T}}=\tau_{\mathrm{T}, \mathrm{e}}+$ $\tau_{\mathrm{T} \pm}$ is the total optical depth and $\tau_{\mathrm{T}, e}$ is the optical depth due to baryonic electrons.

At $E<E_{\mathrm{sa}}$ a photon index of $\alpha=1$ is usually assumed. This indeed holds for a uniform emission region, as is assumed in this work, and is physically expected in our scenario (ii) for volumetric heating. However, when the particles are heated at a moving front, be it a shock or magnetic reconnection front as may be relevant in our scenario (i), then the time they had to cool is proportional to their distance from that front, so that beyond a thin cooling layer where the minimal $\gamma_{m}$ electrons start cooling the electrons become locally essentially mono-energetic with an energy inversely proportional to their distance from the front. Once the emission becomes optically thick at $E<E_{\mathrm{sa}}$ the location of an optical depth of unity from which the photons reach the observer gets closer to the front as $E$ decreases, corresponding to a higher temperature $T^{\prime} \propto E^{\prime-5 / 8}$ so that altogether the observed spectral slope becomes $I_{E^{\prime}}^{\prime \text { JJ }} \propto E^{\prime 2} T^{\prime} \propto E^{\prime 11 / 8}$ or $\alpha=$ 3/8 (Granot, Piran \& Sari 2000; Granot \& Sari 2002). Once the location of optical depth of unity reaches the thin cooling layer where $k_{B} T^{\prime} \sim \gamma_{m} m_{\mathrm{e}} c^{2}=$ const, the usual $\alpha=1$ photon index is recovered (corresponding to a second break energy $E_{\text {ac }}$, so that $\alpha=1$ at $E<$ $E_{\text {ac }}$ while $\alpha=3 / 8$ at $E_{\text {ac }}<E<E_{\mathrm{sa}}$.)

In the top left-hand panel of Fig. 2, we show the spectrum in the cosmological rest-frame of the central engine for different values of $\epsilon_{\mathrm{e}}$. The spectrum shows a distinct peak at $(1+z) E \approx 200 \mathrm{keV}$, which represents the adiabatically cooled thermal component. The spectrum below and above this peak energy is shaped by fast-cooling synchrotron emission from power-law electrons, as shown by the black dashed line, which peaks at $E=E_{m} \approx 1(1+z)^{-1} \mathrm{MeV}$ for the $\epsilon_{\mathrm{e}}=0.1$ case. For smaller values of $\epsilon_{\mathrm{e}}$ the synchrotron peak moves to smaller energies and the normalization of the non-thermal component with respect to the thermal one declines while producing a distinct thermal bump. On the other hand, larger values of $\epsilon_{\mathrm{e}}$ result in a two-hump spectrum until the non-thermal synchrotron component starts to dominate the spectrum completely.

The spectrum drops off sharply at two characteristic energies. At low energies near $E=E_{\mathrm{sa}} \approx 0.5(1+z)^{-1} \mathrm{keV}$, the synchrotron spectrum becomes self-absorbed resulting in a sharp break. At high energies near $(1+z) E=\Gamma m_{\mathrm{e}} c^{2} \approx 0.2 \mathrm{GeV}$, the emission is suppressed due to $\gamma \gamma$-annihilation. The position of the high-energy spectral break is affected by the leaky-box prescription adopted in this work, as argued in Section 3, and therefore the actual break is expected to occur at a larger energy.
In the top right-hand panel of Fig. 2, we show the spectral slopes by plotting $d \log E L_{E} / d \log E$, where the peak (or local minima/maxima) of the spectra occurs when the different curves cross zero. At energies just above $E_{\mathrm{sa}}$, the spectrum is dominated by fast-cooling synchrotron emission, and therefore has the expected slope with $L_{E} \propto E^{-1 / 2}$. Closer to the $E L_{E}$ peak, the spectrum deviates from this trend and becomes harder below the peak and softer above it. This is due to the predominance of the thermal component. However, the peak is not as hard as expected for a Wien spectrum $\left(E L_{E} \propto E^{4}\right)$, the initial condition here. Instead, the spectral slope just below the peak is much softer and remains below unity which is observed for a large fraction of GRBs (e.g. Kaneko et al. 2006). At larger energies above the peak, the synchrotron component again tends to dominate for which $L_{E} \propto E^{-p / 2}$ when $E>E_{m}$. In our model, the value of $p$ depends on the magnetization $\sigma$ according to equation (9) and evolves over time, approaching $p=4$ near the end of dissipation at $r=r_{\mathrm{s}}$.

The particle distribution for both electrons and positrons at $r=r_{\mathrm{s}}$, just before the injection of power-law electron ceases, is shown in the bottom left-hand panel of Fig. 2 as a function of the dimensionless momentum $p_{\mathrm{e}}=\gamma_{\mathrm{e}} \beta_{\mathrm{e}}$. Since $\xi=0.2$ here, the colder baryonic electrons dominate the Thomson optical depth of the flow. However, for larger values of $\epsilon_{\mathrm{e}}$, the fraction of produced $e^{ \pm}$-pairs increases and starts to dominate the optical depth. Starting at high momentum, for $p_{\mathrm{e}}>\gamma_{m}$, with $\gamma_{m}>100$, the curves reflect the distribution of the injected power-law electrons that cools via synchrotron emission. The distribution of cooled electrons at $10 \lesssim p_{\mathrm{e}}<\gamma_{m}$ reflects their steady-state distributed due to cooling, where the differential number of particles at a given $\gamma_{\mathrm{e}}$ reflects the cooling time at that $\gamma_{\mathrm{e}}$, such that $\mathrm{d} n=\gamma_{\mathrm{e}} \mathrm{d} n / \mathrm{d} \gamma_{\mathrm{e}}=\gamma_{e} n_{\mathrm{e}}\left(\gamma_{\mathrm{e}}\right) \propto t_{c}\left(\gamma_{\mathrm{e}}\right) \propto \gamma_{\mathrm{e}}^{-1}$ that yields $n_{e}\left(\gamma_{\mathrm{e}}\right) \propto \gamma_{\mathrm{e}}^{-2}$. In momentum space, $n_{\mathrm{e}}\left(p_{\mathrm{e}}\right)=\left(d \gamma_{\mathrm{e}} / d p_{\mathrm{e}}\right) n_{\mathrm{e}}\left(\gamma_{\mathrm{e}}\right)=\left(p_{\mathrm{e}} / \gamma_{\mathrm{e}}\right) n_{\mathrm{e}}\left(\gamma_{\mathrm{e}}\right)$, and therefore $n_{e}\left(p_{\mathrm{e}}\right) \propto p_{\mathrm{e}} / \gamma_{\mathrm{e}}^{3}$. For $p_{e} \gg 1, p_{\mathrm{e}} \approx \gamma_{\mathrm{e}}$ and so $n_{\mathrm{e}}\left(p_{\mathrm{e}}\right) \propto \gamma_{\mathrm{e}}^{-2}$ and $d n_{\mathrm{e}} / d \log p_{\mathrm{e}} \propto \mathrm{d} \tau_{T} / \mathrm{d} \log p_{\mathrm{e}} \propto p_{\mathrm{e}}^{-1}$. At low $p_{\mathrm{e}}<1$, the particle distribution is a Maxwellian that represents the initial colder baryonic electrons as well as the cooled injected power-law electrons and the produced $\mathrm{e}^{ \pm}$-pairs. Energy exchange between the cooler baryonic electrons and the injected power-law electrons and produced $e^{ \pm}$-pairs occurs via Coulomb scattering, which is included in the numerical code. For larger values of $\epsilon_{e}$, the mean energy of incoming power-law electrons is also larger, which results in the respective Maxwellian distribution having a larger temperature.

For smaller values of $\epsilon_{\mathrm{e}}<0.1$, the total optical depth is dominated by the baryonic electrons as shown in the bottom-right panel of Fig. 2. When $\epsilon_{\mathrm{e}}$ is increased, more energy is put into the non-thermal component that results in increasing the number of produced $\mathrm{e}^{ \pm}$pairs, as evident for the $\epsilon_{\mathrm{e}}=0.2$ case. Due to pair production the photospheric radius is extended to slightly larger radii by a factor $\left(1+\tau_{\mathrm{T}, \pm} / \tau_{\mathrm{T}, \mathrm{e}}\right)^{3 / 5}$ over the baryonic one given in equation (6). For example, $\tau_{\mathrm{T}, \pm} \approx \tau_{\mathrm{T}, \mathrm{e}}$ for $\epsilon_{\mathrm{e}}=0.2$ that yields an enhancement in the photospheric radius by a factor $\sim 2^{3 / 5} \approx 1.52$. This is demonstrated in the figure where the dotted black line shows the radial evolution of the optical depth in the absence of pair-production, and the solid lines show the total optical depth including $\mathrm{e}^{ \pm}$-pairs. After a surge in $\tau_{\mathrm{T}}$ due to the produced pairs, the solid lines display similar radial evolution as compared to the black dotted line that follows $\tau_{\mathrm{T}} \propto r^{-5 / 3}$ for $r<r_{\mathrm{s}}$ and $\tau_{\mathrm{T}} \propto r^{-1}$ for $r>r_{\mathrm{s}}$. For all the cases, the Compton- $y$ parameter, $y_{C}=(4 / 3)\left(\left\langle\gamma_{\mathrm{e}}^{2}\right\rangle-1\right) \tau_{\mathrm{T}}$, which measures the importance of Compton scattering, remains smaller than unity since the mean energy of the particles is dominated by the cooler baryonic electrons. As we discuss below, particles in this scenario mainly cool via synchrotron emission and Compton scattering is not important.

In the left-hand panel of Fig. 3, we show the spectrum for different values of $\xi$, which sets the fraction of the injected electrons 

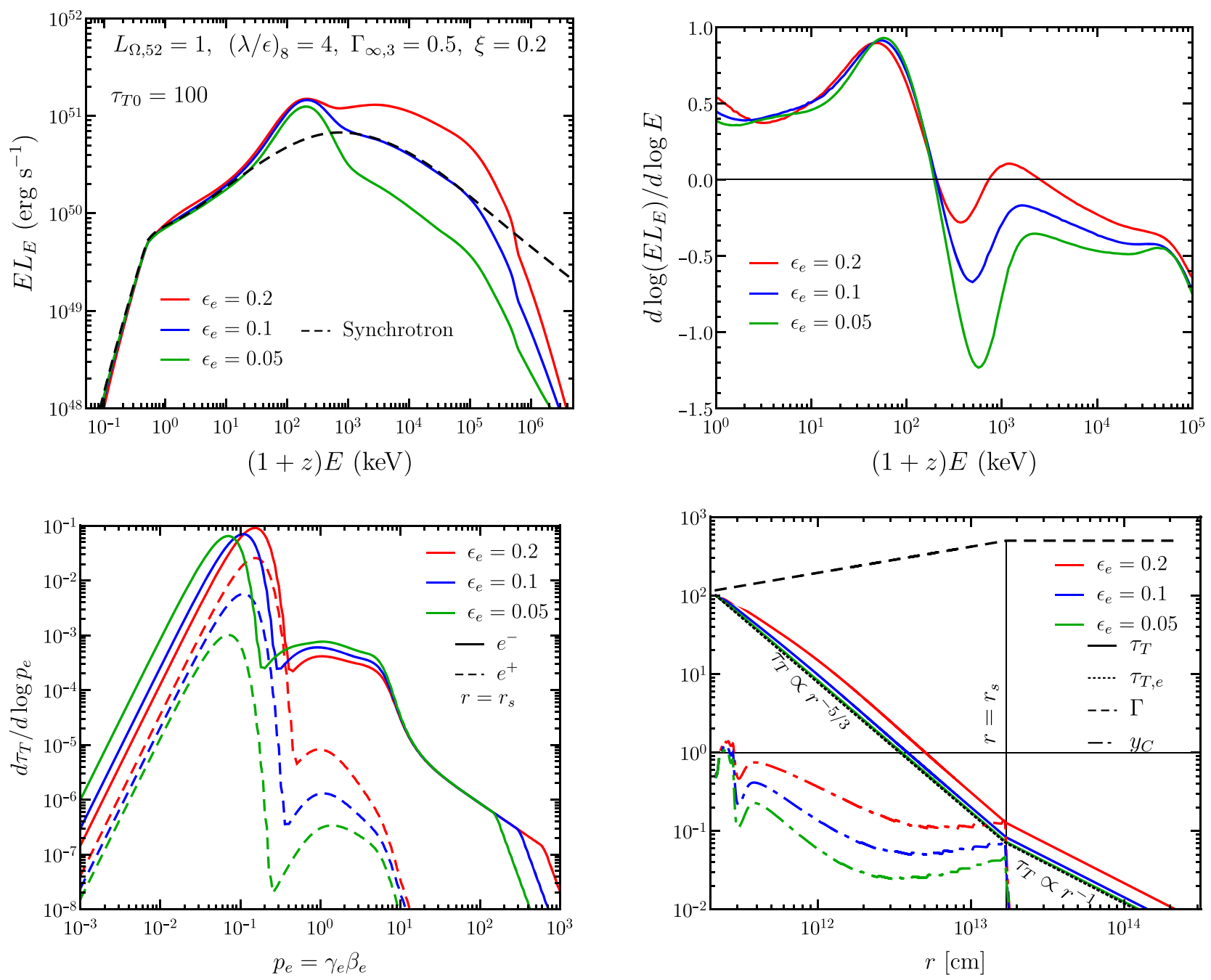

Figure 2. Top left: Observed steady-state spectrum with injection of power-law electrons commencing at $\tau_{T 0}=100$. The final spectrum is obtained when the flow becomes optically thin with $\tau_{\mathrm{T}} \ll 1$. The black dashed line shows the synchrotron emission from power-law electrons (without the effects of $\gamma \gamma$-annihilation at the highest energies). Top right: Spectral slopes where the photon index $\alpha=-2+\operatorname{dlog}\left(E L_{E}\right) / d \log E$. Bottom left: Electron and positron momentum distributions at $r=r_{\mathrm{s}}$ shown using the optical depth. Bottom right: Radial evolution of the bulk LF $\Gamma$, total optical depth of $\tau_{\mathrm{T}}=\tau_{\mathrm{T}, \mathrm{e}}+\tau_{\mathrm{T}, \pm}$ including that due to produced $e^{ \pm}$-pairs $\left(\tau_{T, \pm}\right)$, optical depth of baryonic electrons only $\left(\tau_{\mathrm{T}, \mathrm{e}}\right)$ if no pairs were produced, and Compton-y parameter of pairs $\left(y_{C}\right)$. Magnetic energy dissipation and acceleration of the flow halts at the saturation radius $r=r_{\mathrm{s}}$, beyond which the flow coasts at constant $\Gamma=\Gamma_{\infty}$. The photospheric radius due to baryonic electrons $\left(\tau_{T, \mathrm{e}}=1\right)$ is extended due to production of $e^{ \pm}$-pairs $\left(\tau_{T}=1\right)$.

accelerated into a power law. As a result, $\xi$ affects the mean energy of power-law electrons and consequently $\gamma_{m}$, where both are inversely proportional to $\xi$. The effect of decreasing $\xi$ is similar to that of increasing $\epsilon_{\mathrm{e}}$. Since the number of electrons injected into the emission region remains fixed, increasing the mean energy of the distribution also increases the contribution of the non-thermal synchrotron component. Consequently, the optical depth due to pair production also increases with increasing $\xi$.

The right-hand panel of Fig. 3 shows the effect on the spectrum when the power-law index $p$ of incoming electrons is fixed rather than left to vary with the magnetization, as assumed in the model here in equation (9). As the value of $p$ is lowered, the synchrotron spectrum at $E>E_{m}$ becomes harder since $L_{E} \propto E^{-p / 2}$. By using 2D and 3D PIC simulations, Sironi \& Spitkovsky (2014) find that $p$ $\gtrsim 1.5$ for $\sigma \lesssim 50$, which means that the synchrotron spectrum can become even harder than shown in the figure if $\sigma$ is larger in the emission region. Indeed, this type of spectrum with a quasi-thermal peak and a hard power-law component has been observed in, e.g., GRB 090902B (Abdo et al. 2009). This type of scenario can also explain the observation of a $31.5[(1+z) / 2.82] \mathrm{GeV}$ photon in the central-engine frame in this GRB during the prompt emission since the hard synchrotron spectrum extends to $\mathrm{GeV}$ energies.

\subsubsection{Radial evolution of the spectrum and particle distribution}

We present the radial evolution of the spectrum, the corresponding particle distribution, and flow parameters for the case with $\epsilon_{\mathrm{e}}=0.1$ in Fig. 4. The spectrum is obtained for different optical depths, as shown by the black dots on the red curve in the bottom panel of Fig. 4, and correspondingly different radii where we integrate the comoving emissivity over radial extent $\Delta r / r=1 / 2$ centred on the radius corresponding to the chosen $\tau_{\mathrm{T}}$. The observed steady-state spectrum, shown by the black dashed line, is effectively a sum over the optically thin spectra where the radial integration of the comoving 

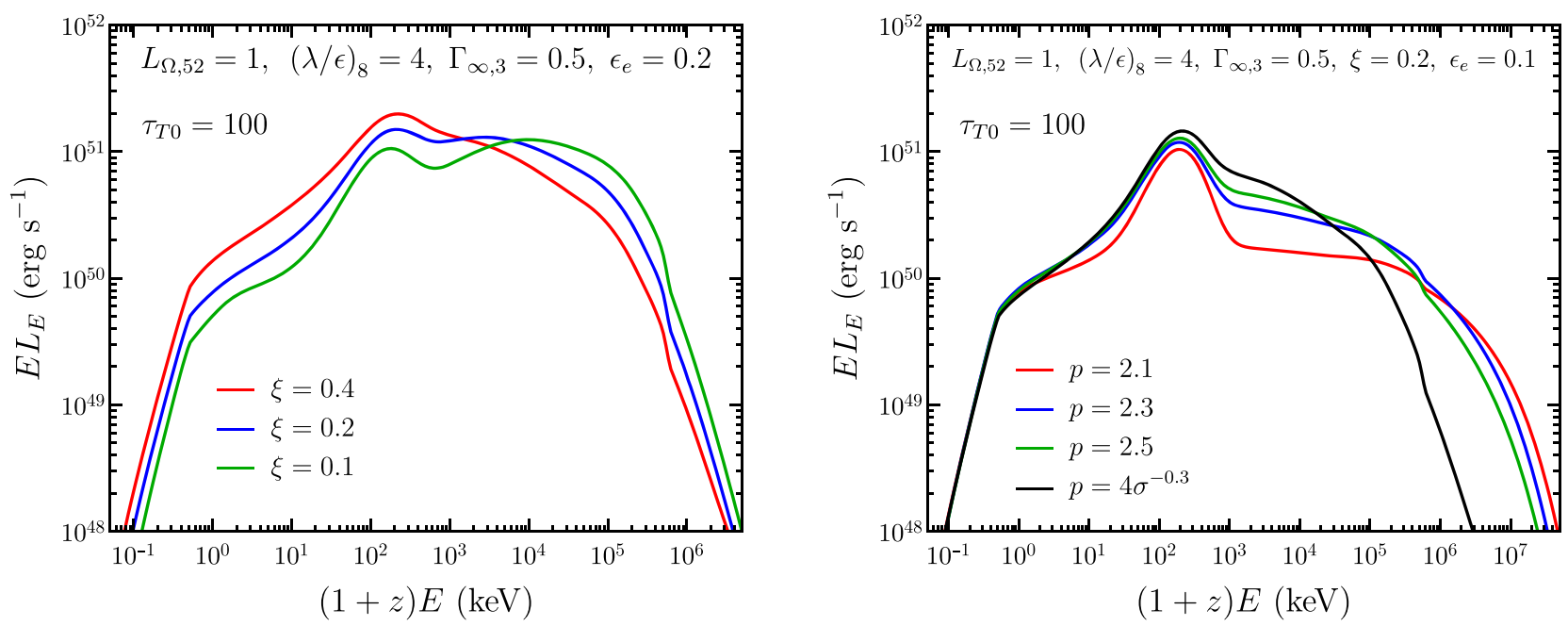

Figure 3. Left: Observed steady-state spectrum from the injection of power-law electrons for different fraction $\xi$ of total incoming electrons accelerated in magnetic reconnection layers. Right: Spectrum for different power-law index $p$ of the injected electrons with energy distribution $n^{\prime}\left(\gamma_{\mathrm{e}}\right) \propto \gamma_{\mathrm{e}}^{-p}$.

emissivity is performed for $r>r_{\mathrm{ph}}(\tilde{\theta})$. At early times, the spectrum is dominated by the initial condition given by the Wein-like spectrum from equation (14). Injection of power-law electrons gives rise to the fast-cooling synchrotron spectrum, which builds up over time while the thermal peak cools and dilutes due to adiabatic expansion of the outflow. After the flow becomes optically thin $\left(\tau_{\mathrm{T}}<1\right)$, the thermal peak starts to shift to higher energies since the radiation field is no longer adiabatically cooled and the thermal peak is simply blueshifted to higher energies by the increasing $\Gamma$ from its value attained in the comoving frame at $\tau_{\mathrm{T}}=1$. High-energy spectrum at energies $E>\Gamma m_{\mathrm{e}} c^{2} /(1+z)$ is suppressed due to $\left(\gamma \gamma \rightarrow \mathrm{e}^{ \pm}\right)$pair-production. The produced $\mathrm{e}^{ \pm}$-pairs annihilate and yield a sharply peaked spectral component at $E=\Gamma m_{\mathrm{e}} c^{2} /(1+z)$ at very early times.

In this case $\xi=0.2$, and therefore the initial optical depth is dominated by thermal baryonic electrons. However, copious pair production ensues after the injection of power-law electrons and $\mathrm{e}^{ \pm}$pairs start to become comparable to the baryonic electrons in optical depth. The injection of power-law electrons also raises the mean energy per particle $\left\langle\gamma_{\mathrm{e}}\right\rangle$, as can be seen from the rightward shift of the peak of the thermal particle distribution in the middle panel of Fig. 4 as well as from the radial evolution of $\left\langle\gamma_{\mathrm{e}}\right\rangle-1$ shown in the bottom panel. However, the Compton- $y$ parameter remains below unity as the rate of heating is insufficient to make Comptonization important. The cooled power-law electrons as well as the produced pairs ultimately join the thermal distribution.

The power-law electrons cool primarily due to synchrotron emission. This can be understood by comparing the magnetic field energy density to that of the thermal radiation field. For $r>r_{\tau 0}$, where $r_{\tau 0}$ is the radius corresponding to $\tau_{\mathrm{T} 0}$ when injection of power-law electron commences, the comoving energy density of the thermal component is $U_{\mathrm{th}}^{\prime}(r)=U_{0}^{\prime}\left(r / r_{\tau 0}\right)^{-28 / 9}=U_{0}^{\prime} \tau_{T 0}^{-28 / 15}\left(r / r_{\mathrm{ph}}\right)^{-28 / 9}$ since the injected energy is no longer completely thermalized. Therefore, the thermal component simply adiabatically cools for $r>r_{\tau 0}$. The initial energy density is given by $U_{0}^{\prime} \approx\left(4 \sigma_{\mathrm{SB}} / c\right)\left[T_{\mathrm{ph}}^{\prime}\left(r_{\tau 0} / r_{\mathrm{ph}}\right)^{-7 / 12}\right]^{4}=$ $\left(4 \sigma_{\mathrm{SB}} / c\right) T_{\mathrm{ph}}^{\prime 4} \tau_{T 0}^{7 / 5}$. The energy density of the magnetic field is given by $U_{B}^{\prime}=B^{\prime 2} / 8 \pi$, which then yields

$\frac{U_{B}^{\prime}}{U_{\mathrm{th}}^{\prime}}=69 \frac{\Gamma_{\infty, 3}\left(\frac{\lambda}{\epsilon}\right)_{8}^{1 / 5}}{L_{\Omega, 52}^{1 / 5} \tau_{\mathrm{T}, \mathrm{e}}^{4 / 15}}$, indicating that power-law electrons mainly cool by synchrotron emission. In addition, Compton cooling of injected electrons is suppressed as it occurs in the Klien-Nishina regime for photons with energy above

$E=\frac{\Gamma}{(1+z) \gamma_{m}} m_{\mathrm{e}} c^{2}=171\left(\frac{\xi}{\epsilon_{e}}\right) \frac{r_{12}^{2 / 3}}{\Gamma_{\infty, 3}^{1 / 3}\left(\frac{\lambda}{\epsilon}\right)_{8}^{2 / 3}} \mathrm{keV}$,

which suggests that the non-thermal synchrotron component above the thermal peak cannot cool the power-law electrons by inverse Compton scattering.

The injected energy density at a given radius $\tilde{r}$ is reduced as the flow expands adiabatically, such that $\mathrm{d} U_{\text {inj }}^{\prime}(r)=\mathrm{d} U_{\text {inj }}^{\prime}(\tilde{r})(\tilde{r} / r)^{-28 / 9}$, where the injected energy density between $\tilde{r}$ and $\tilde{r}+\mathrm{d} \tilde{r}$ is $\mathrm{d} U_{\text {inj }}^{\prime}(\tilde{r})=$ $\left(\epsilon_{e} / 2\right)\left[\mathrm{d} U_{\mathrm{diss}}^{\prime}(\tilde{r}) / \mathrm{d} t^{\prime}\right] \mathrm{d} \tilde{r} / \Gamma c$. The total injected energy density surviving at $r \gg r_{\text {inj }}$, where $r_{\text {inj }}$ is the radius where energy injection commences, is obtained by integrating over $\tilde{r}$ that yields

$U_{\text {inj }}^{\prime}(r)=\frac{3}{7} \frac{\epsilon_{\mathrm{e}} L_{\Omega}}{c \Gamma_{\infty}} \frac{1}{r^{2} \Gamma}=\frac{9}{7} \epsilon_{\mathrm{e}} \frac{\mathrm{d} U_{\mathrm{diss}}^{\prime}}{\mathrm{d} t^{\prime}} \frac{r}{\Gamma c}$.

The non-thermal emission will begin to dominate the thermal component when $U_{\mathrm{th}}^{\prime} / U_{\mathrm{inj}}^{\prime}<1$, where

$\frac{U_{\text {th }}^{\prime}}{U_{\text {inj }}^{\prime}}=4 \times 10^{-2} \frac{L_{\Omega, 52}^{7 / 15}\left(\frac{\lambda}{\epsilon}\right)_{8}^{14 / 45}}{\epsilon_{e} \Gamma_{\infty, 3}^{7 / 9} r_{12}^{7 / 9}}$.

For the fiducial parameters chosen in Fig. 4, the above condition is not satisfied before dissipation ceases, and therefore the non-thermal synchrotron component never fully dominates over the thermal component. The above estimate is strictly valid when the flow is optically thick for which the radiation field energy density follows the scaling $U_{\mathrm{th}}^{\prime} \propto r^{-28 / 9}$. Adiabatic cooling of the radiation field stops once the flow becomes optically thin, at which point it only suffers density dilution due to the volume expansion but no cooling.

\subsection{Distributed Heating of Particles}

Earlier we explored the scenario where a fraction of the incoming baryonic electrons are directly accelerated into a power-law energy distribution at magnetic reconnection sites. Here, we consider an alternative, where magnetic energy dissipation in the flow, e.g. 

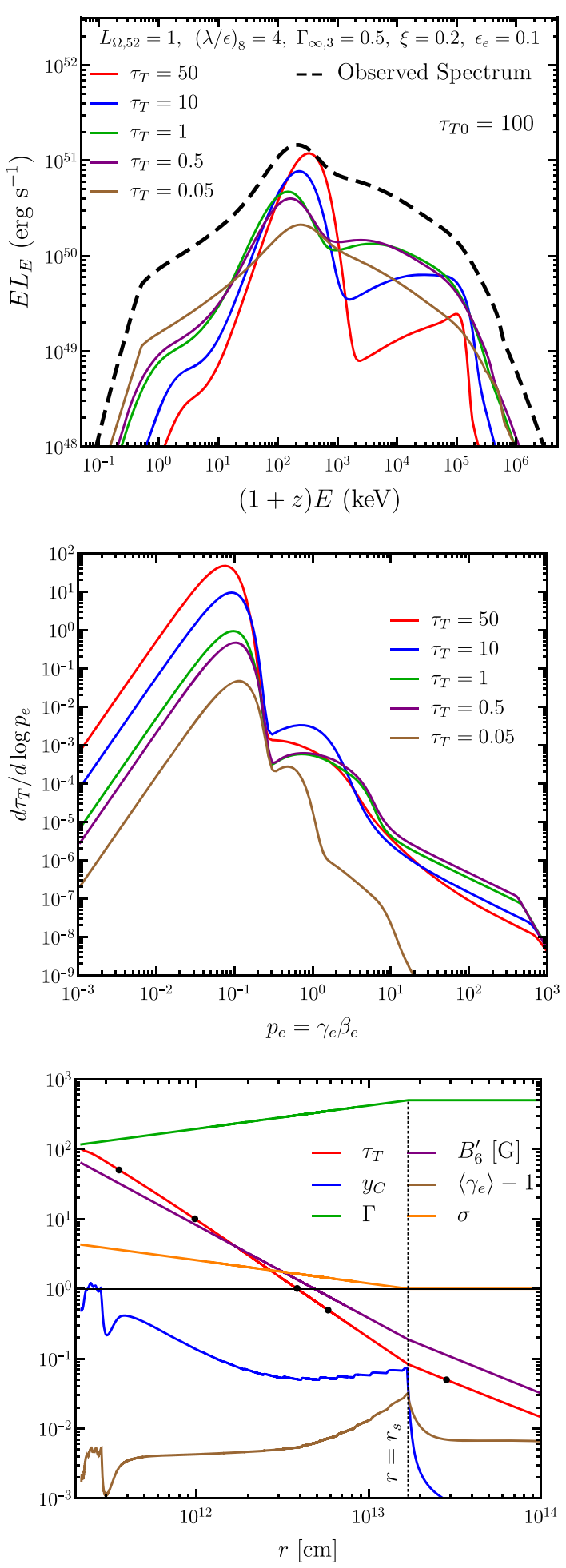

Figure 4. Top: Evolution of the spectrum sampled at different total optical depth $\tau_{\mathrm{T}}$ that was emitted over half a dynamical time $(\Delta r / r=1 / 2)$ centred at the radius corresponding to $\tau_{T}$. The observer only sees the final spectrum, shown using a dashed black line, which is effectively a sum over the optically thin spectra with emission arising from $r>r_{\mathrm{ph}}(\tilde{\theta})$. Middle: Evolution of particle distribution that remains dominated by the initial thermal component since $\xi=0.2$ in this case. Bottom: Radial evolution of flow parameters with black dots, marking the optical depth $\tau_{\mathrm{T}}$ for which the spectra is shown in the top panel. due to MHD instabilities, leads to distributed heating of all electrons (Thompson 1994; Ghisellini \& Celotti 1999; Giannios 2006; Giannios \& Spruit 2007; Giannios 2008). The comoving energy dissipation rate per unit volume, $\mathrm{d} U_{\text {diss }}^{\prime} / \mathrm{d} t^{\prime}$, is given in equation (8) out of which only a fraction $\epsilon_{e} / 2$ goes into heating the electrons in the emission region, such that the volumetric heating rate is $\mathrm{d} U_{e}^{\prime} / \mathrm{d} t^{\prime}=$ $\left(\epsilon_{\mathrm{e}} / 2\right) \mathrm{d} U_{\text {diss }}^{\prime} / \mathrm{d} t^{\prime}$. Deeper in the flow, at larger optical depths $\tau_{T} \gg 1$, the thermal radiation field is the dominant coolant (see equation 18). The continuous heating and simultaneous cooling of particles drives their energy distribution to peak at a critical temperature at which point heating is balanced by cooling. The Compton cooling rate per unit volume for a thermal electron distribution is given by

$\frac{\mathrm{d} U_{c}^{\prime}}{\mathrm{d} t^{\prime}}=4 n_{\mathrm{e}}^{\prime}\left(\frac{k_{B} T_{\mathrm{e}}^{\prime}}{m_{e} c^{2}}\right) \sigma_{\mathrm{T}} c U_{\mathrm{th}}^{\prime}$,

where again we make the simplifying assumption that approximately half of the dissipated energy goes directly towards accelerating the flow and the remaining half converts to the thermal radiation field with energy density $U_{\text {th }}^{\prime}$. By equating the cooling rate to that of particle heating, $d U_{c}^{\prime} / d t^{\prime}=d U_{e}^{\prime} / d t^{\prime}$, we find the critical temperature at which particles congregate

$k_{B} T_{\mathrm{e}, \text { crit }}^{\prime}=138 \frac{\epsilon_{\mathrm{e}} \Gamma_{\infty, 3}^{5 / 3} r_{12}^{5 / 3}}{L_{\Omega, 52}\left(\frac{\lambda}{\epsilon}\right)_{8}^{2 / 3}} \mathrm{keV} \approx 132 \frac{\epsilon_{\mathrm{e}}}{\tau_{T, \mathrm{e}}} \mathrm{keV}$.

This temperature is smaller at smaller radii or at larger optical depths, however, it cannot become smaller than that of the thermal radiation field. Therefore, below an equilibrium radius or above the optical depth (Giannios 2006),

$$
\begin{aligned}
r_{\text {eq }} & =5 \times 10^{10} \frac{L_{\Omega, 52}^{5 / 9}\left(\frac{\lambda}{\epsilon}\right)_{8}^{1 / 3}}{\epsilon_{\mathrm{e}}^{4 / 9} \Gamma_{\infty, 3}^{8 / 9}} \mathrm{~cm} \\
\tau_{\text {eq }} & =133 \frac{\epsilon_{\mathrm{e}}^{20 / 27} L_{\Omega, 52}^{2 / 27}\left(\frac{\lambda}{\epsilon}\right)_{8}^{1 / 9}}{\Gamma_{\infty, 3}^{5 / 27}},
\end{aligned}
$$

radiation and particles are in thermal equilibrium. Above that radius, electrons fall out of equilibrium and attain a higher effective temperature (since the distribution becomes narrowly peaked and does not remain Maxwellian) as compared to the thermal radiation field. The details of how distributed heating is implemented in the simulation are presented in the Appendix.

As the flow expands, the energy density of the thermal radiation field declines. This increases the time-scale over which particles are cooled by Comptonization. Particles are also cooling due to adiabatic expansion, the time-scale for which is (see the Appendix) $t_{\mathrm{ad}}^{\prime}=(3 / 7) r / \Gamma c \propto r^{2 / 3}$ for $r<r_{\mathrm{s}}$. The Compton cooling timescale is $t_{c}^{\prime}=3 m_{\mathrm{e}} c / 4 \sigma_{T} \gamma_{\mathrm{e}} U_{\mathrm{th}}^{\prime}$, for particles with $\mathrm{LF} \gamma_{\mathrm{e}}$, where $U_{\text {th }}^{\prime}=U_{0}^{\prime}\left(r / r_{\tau 0}\right)^{-28 / 9}$ and $r_{\tau 0}$ is the radius corresponding to $\tau_{T 0}$ where heating of particles commences. Comparison of the two time-scales yields

$\frac{t_{c}^{\prime}}{t_{\mathrm{ad}}^{\prime}}=\frac{7}{4} \frac{m_{\mathrm{e}} c^{2}}{\sigma_{T} U_{\mathrm{th}}^{\prime} \gamma_{\mathrm{e}}} \frac{\Gamma}{r} \approx \frac{5 \times 10^{-2}}{\gamma_{\mathrm{e}}} \frac{r_{12}^{22 / 9} \Gamma_{\infty, 3}^{22 / 9}}{L_{\Omega, 52}^{22 / 15}\left(\frac{\lambda}{\epsilon}\right)_{8}^{44 / 45}}$,

which suggest that particles cool predominantly via Comptonization.

In the top left-hand panel of Fig. 5, we present the observed steady-state spectrum for a heated flow for different values of $\epsilon_{\mathrm{e}}$. For the chosen fiducial parameters, $\tau_{\text {eq }} \approx 32$, and therefore the condition for thermal equilibrium holds for $\tau_{\mathrm{T} 0}=100$, the initial baryonic electron Thomson optical depth at which the simulation is initialized. In all cases, due to multiple Compton scattering, the spectrum extends smoothly to high energies above the adiabatically cooled thermal peak that appears at $(1+z) E \sim 1 \mathrm{MeV}$. This energy 

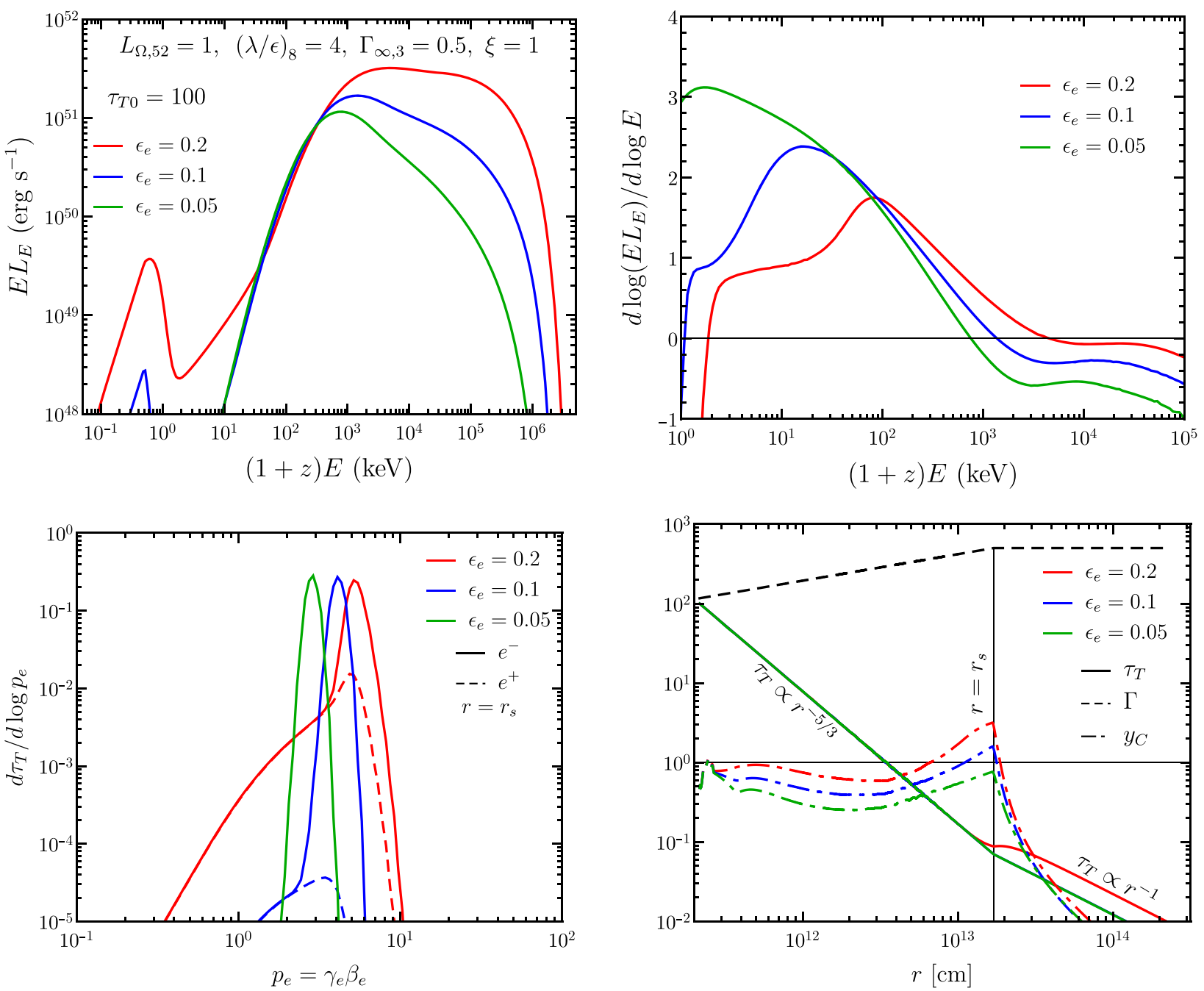

Figure 5. Top left: Observed steady-state spectrum with distributed heating of particles commencing at $\tau_{\mathrm{T} 0}=100$. The final spectrum is obtained at $\tau_{\mathrm{T}} \ll 1$ when the flow is optically thin. For the chosen fiducial parameters the equilibrium optical depth is $\tau_{\text {eq }} \approx 32$. Top right: Spectral slopes with the photon index given by $\alpha=-2+\mathrm{d} \log \left(E L_{E}\right) / \mathrm{d} \log E$. Bottom left: Electron and positron particle distribution at $r=r_{\mathrm{s}}$, the radius at which the mono-energetic distribution are expected to be the hottest. Bottom right: Evolution of flow parameters with radius.

is higher due to Comptonization than that expected from adiabatic cooling which freezes at the photospheric radius. These results are consistent with that shown in Giannios (2006), Giannios \& Spruit (2007), Giannios (2008) who used a Monte Carlo code without pair cascade effects. The effect of increasing $\epsilon_{\mathrm{e}}$ is to put more energy into the non-thermal Comptonized spectral component and to make the spectrum harder above the thermal peak. In addition, for larger $\epsilon_{\mathrm{e}}$ a pronounced peak around $(1+z) E \approx 0.5 \mathrm{keV}$ develops due to selfabsorbed synchrotron emission from mildly relativistic electrons.

The low-energy spectral index, as shown in the top right-hand panel of Fig. 5, becomes softer with increasing $\epsilon_{e}$. It only approaches the photon index $\alpha=-2+\operatorname{dlog}\left(E L_{E}\right) / \operatorname{dlog} E \sim-1$ typically observed in prompt GRB emission for $\epsilon_{\mathrm{e}} \gtrsim 0.2$, and below that the low-energy spectrum appears to be too hard. This can be understood by looking at the evolution of the Compton-y parameter shown in the bottom right-hand panel of Fig. 5. For larger $\epsilon_{\mathrm{e}}, y_{C}$ is also larger and substantially exceeds unity in the $\epsilon_{\mathrm{e}}=0.2$ case. This results in the Comptonization of the softer synchrotron photons towards the thermal peak which softens the low-energy spectrum. For smaller $\epsilon_{e}, y_{C}$ remains below unity and the soft synchrotron photons are not efficiently Comptonized to higher energies, leading to harder low-energy spectral slopes.

The optical depth in all cases remains unaltered from the trend expected for baryonic electrons, which suggests that pair-production is mostly insignificant in the cases shown here. This can also be seen in the bottom left-hand panel of Fig. 5 where the optical depth is dominated by baryonic electrons. Only for $\epsilon_{\mathrm{e}}=0.2$, copious pair-production ensues at $r \sim r_{\mathrm{s}}$ as the high-energy spectrum exceeds the pair-production threshold due to $y_{C}>1$. The particle distributions are sharply peaked at the momentum, where heating and cooling of particles are in balance. This is in stark contrast with the particle distribution in the scenario with power-law electron injection.

Since $e^{ \pm}$-pairs are subdominant and the optical depth is dominated by the baryonic electrons, the dimensionless momentum of electrons, $p_{\mathrm{e}}=\gamma_{\mathrm{e}} \beta_{\mathrm{e}}=\left(\gamma_{\mathrm{e}}^{2}-1\right)^{1 / 2}$, at which they congregate after the heating commences at $r>r_{\tau 0}$, can be obtained by comparing their heating and Compton cooling rates. The cooling rate for a mono-energetic distribution is $\mathrm{d} U_{c}^{\prime} / \mathrm{d} t^{\prime}=(4 / 3) \sigma_{T} c p_{\mathrm{e}}^{2} n_{\mathrm{e}}^{\prime} U_{\text {th }}^{\prime}$, where $U_{\text {th }}^{\prime}=U_{0}^{\prime}\left(r / r_{\tau 0}\right)^{-28 / 9}$ is the energy density of the adiabatically cooled thermal radiation. 
Then, heating and cooling balance yields

$p_{e}(r)=2.6 \frac{\epsilon_{e}^{1 / 2} \Gamma_{\infty, 3}^{11 / 9} r_{12}^{11 / 9}}{L_{\Omega, 52}^{11 / 15}\left(\frac{\lambda}{\epsilon}\right)_{8}^{22 / 45}}=2.6 \frac{\epsilon_{e}^{1 / 2}}{\tau_{T, e}^{11 / 15}}$.

Note the above estimate is different from that derived in equation (23) in two respects. First, it assumes monoenergetic particles and not a Maxwellian distribution, where the former is relevant here. Secondly, for the cooling rate it assumes the adiabatically cooled energy density of the radiation field normalized at $r=r_{\tau 0}$, whereas the scaling of $U_{\text {th }}^{\prime} \propto r^{-7 / 3}$ is assumed for equation (23) since the energy given to particles is assumed to be completely thermalized, which results in a shallower decay profile for the radiation field energy density. This estimate for $p_{\mathrm{e}}$ strictly assumes that $e^{ \pm}$-pairs are subdominant, but they may become important for some model parameters in which case the above estimate will not hold. For the fiducial parameters in Fig. 5, the electrons attain a maximum $p_{e, \max } \simeq 5$ at $r=r_{\mathrm{s}}$ when $\epsilon_{\mathrm{e}}=0.1$, as shown in the bottom left-hand panel of the figure.

The radial evolution of the spectrum and particle distribution, along with that of the flow parameters, for the case of $\epsilon_{\mathrm{e}}=0.1$ is presented in Fig. 6. The initial spectrum at large optical depths is dominated by the thermal component that adiabatically cools and dilutes as the flow expands. Meanwhile, continuous dissipation in the flow heats up the baryonic electrons, as evident from the rightward shift of the narrowly peaked particle distribution (middle panel) as well as from the rising $\left\langle\gamma_{\mathrm{e}}\right\rangle-1$ (bottom panel). This leads to gradual broadening of the Wien distribution as well as the shift of the thermal peak to higher energies. The high-energy spectrum only develops when the flow has become sufficiently optically thin (also see e.g. fig. 1 of Giannios 2008). Heating of the flow terminates at $\tau_{\mathrm{T}}=$ 0.08 , and therefore, the particle distribution at $\tau_{\mathrm{T}}=0.05$ lacks a sharp peak that is expected due to the balance between heating and cooling. Notice that the particle distribution shown in the middle panel of Fig. 6 is the instantaneous distribution at a given $\tau_{\mathrm{T}}$, and the corresponding spectrum is integrated over $\Delta r / r=1 / 2$ while centred at the radius corresponding to the chosen $\tau_{\mathrm{T}}$.

A consequence of mildly relativistic mono-energetic electrons is the emergence of a self-absorbed cyclo-synchrotron peak at $(1+z) E \approx 0.5 \mathrm{keV}$. In the earlier scenario of power-law electron injection, the spectrum showed a break around the same energy rather than a narrow peak. This signature is a potential discriminant between the two scenarios, and prompt GRB observations in soft Xrays should be able to distinguish between the two particle heating mechanisms.

\section{PARAMETER SPACE STUDY}

We consider here different outflow parameters to see their effect on the final spectrum. From equations (3) and (6), we see that a change in the model parameters is reflected in the relative position of the saturation radius with respect to the photospheric radius, which is parametrized by their ratio,

$\kappa \equiv \frac{r_{s}}{r_{\mathrm{ph}}}=17 \frac{\Gamma_{\infty, 3}^{3}\left(\frac{\lambda}{\epsilon}\right)_{8}^{3 / 5}}{L_{52}^{3 / 5}}$.

Therefore, it is more intuitive to scale a given model parameter, while the other two parameters are kept fixed, using the ratio $\kappa$ with a clear expectation. If $\kappa$ is much larger than unity, a large fraction of the dissipated energy will be injected into the emission region when it is optically thin. This will result in a pre-dominantly nonthermal spectrum. On the other hand, when $\kappa \approx 1$, the spectrum
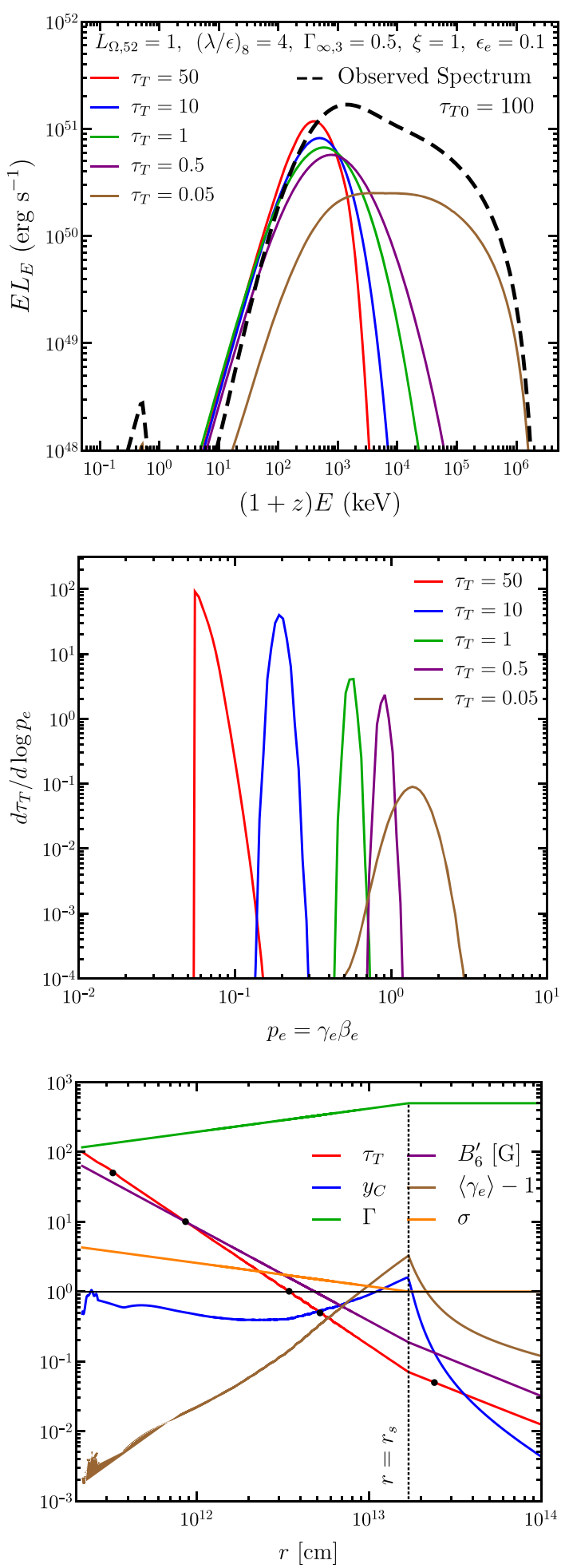

Figure 6. Top: Evolution of the spectrum with radius for the distributed heating scenario, obtained at different optical depths $\tau_{T}$ where the spectrum was emitted within half a dynamical time around the radius corresponding to $\tau_{T}$. The observed spectrum, shown using a black dashed line, is effectively a sum over the optically thin spectra with emission arsing from $r>r_{\mathrm{ph}}(\tilde{\theta})$. Middle: The corresponding particle distribution that remains sharply peaked at the critical momentum where particle heating and cooling are balanced. Bottom: Evolution of flow parameters. Black dots mark the optical depth for which spectra and particle distribution are shown. 
should have a pronounced quasi-thermal component since most of the energy was dissipated below the photosphere that led to its thermalization.

In Fig. 7, we present a survey of the model parameter space by showing the spectra for different values of $\kappa$ for the two particle heating scenarios. For both scenarios, the $\kappa=1$ spectrum is dominated by a quasi-thermal component, albeit with low-energy spectral slope much softer than a pure thermal spectrum. The spectrum in the distributed heating scenario is particularly interesting as it shows a more narrowly peaked spectral component. This type of spectra have been observed in a small number of GRBs, e.g. GRB 990123 (Briggs et al. 1999), GRB 090902B (Abdo et al. 2009), and GRB 130427A (Ackermann et al. 2014), which would suggest that dissipation in these bursts was mostly sub-photospheric with minimal energy dissipated in the optically thin parts of the flow. The typical spectrum of GRBs is non-thermal that can be explained when $\kappa>1$ and for which significant fraction of the energy is dissipated in the optically thin parts of the flow. In some bursts, e.g. GRB 110731A (Ackermann et al. 2013), a time-resolved analysis finds a quasi-thermal spectrum for the initial few pulses followed by nonthermal spectrum for the later pulses. This kind of spectral evolution can be explained if $\kappa \approx 1$ for the initial few pulses that were emitted from a smaller radius compared to the pulses that arrived later with $\kappa$ $>1$ that were emitted from a relatively larger radius (or alternatively corresponding to a larger source variability time, $t_{v} \sim \lambda / c$, or a somewhat larger $\Gamma_{\infty} \approx \sigma_{0}$ ).

The effect of changing the model parameters can be seen by comparing the final spectrum in the different panels. An increase in $\left(\frac{\lambda}{\epsilon}\right)_{8}$, which characterizes the lab-frame width of the magnetic field polarity reversal in a striped wind, with $\epsilon \sim 0.1$, shifts the thermal peak towards lower energies since $E_{\mathrm{pk}, \mathrm{th}}\left(r_{\mathrm{ph}}\right) \propto\left(\frac{\lambda}{\epsilon}\right)_{8}^{-7 / 20}$ Likewise, in the distributed heating scenario, the adiabatically cooled but also Comptonized thermal peak energy becomes softer. When the jet power per unit solid angle $L_{\Omega}$ is varied, the main effect is to increase the overall normalization of the spectrum. In the distributed heating case, the low-energy spectrum below the thermal peak also becomes softer as $\kappa$ is increased. Finally, as the saturation LF $\Gamma_{\infty}$ is increased for $\kappa>1$, the spectrum shows minimal changes above the thermal peak but becomes increasingly softer below the thermal peak for larger $\Gamma_{\infty}$ in both heating scenarios. In addition, the soft $\mathrm{X}$-ray peak becomes more prominent with increasing $\kappa$. In general, larger $\kappa$ tends to produce broader Band function peaks with softer low-energy spectra simply due to the fact that most of the injected energy went into the non-thermal spectral component rather than being thermalized.

\section{SPECTRAL BREAK BELOW THE PEAK}

A spectral break below the $E L_{E}$-peak $\left(E_{\mathrm{pk}}\right)$ has been observed in the time-integrated as well as time-resolved spectra of a small number of bursts. Such cases have been modelled as having two spectral components where the low-energy component is thermal with its peak located at the break energy, $E_{\mathrm{th}}=E_{\mathrm{br}} \sim 100 \mathrm{keV}$, while the high-energy non-thermal component peaks at $E=E_{\mathrm{pk}} \sim 500 \mathrm{keV}$, where both components show fluctuations in the break and peak energies by a factor of $\sim 2$ (or more for the latter) in a timeresolved analysis (Guiriec et al. 2015a). Alternatively, Ravasio et al. $(2018,2019)$ find that a double smoothly broken power-law (2SBPL) function obtains a slightly better fit over a two component thermal plus non-thermal fitting function with $\left\langle E_{\mathrm{br}}\right\rangle \sim 100 \mathrm{keV}$ and $\left\langle E_{\mathrm{pk}}\right\rangle \sim 1 \mathrm{MeV}$. The two spectral profiles do differ in one way. A
2SBPL spectral profile features a power law at energies $E_{\mathrm{br}}<E<$ $E_{\mathrm{pk}}$, whereas a two component model shows separate humps with a slight depression in the middle in some cases depending on the relative normalization of the thermal and non-thermal components. How well can these two spectral profiles be distinguished from each other depends on the quality of the data, but generally both are found to be consistent.

In the left-hand panel of Fig. 8, we show two spectra featuring a low-energy break for the two particle heating scenarios considered in this work. When power-law particles are injected the resulting spectrum features two humps, where the low-energy hump is the thermal component and high-energy hump is the non-thermal synchrotron emission. In this scenario, it would be difficult to get a smoothly connecting spectrum without any depression between the two peaks, unless the synchrotron component completely dominates. In the case of distributed heating of particles, a hard Comptonized spectrum above the thermal peak can result for particle heating efficiency $\epsilon_{e}$ $\gtrsim 0.3$. The spectrum in this case extends more smoothly to high energies, although a slight depression can be present, due to its origin in multiple Compton scatterings. However, the distributed heating scenario produces the second hump that would be identified as the $E L_{E}$-peak, at much higher energies than observed, even after correcting for the source redshift. The problem lies in requiring a significantly high $\left(y_{C} \gtrsim 5\right)$ Compton-y parameter, which for an optically thin flow necessarily demands high particle momenta $p_{\mathrm{e}}$ (see equation 27). As a consequence, the Comptonized hump always appears at large energies.

In the right-panel panel of Fig. 8, we show the spectral slopes. For the power-law injection case, the low-energy spectrum below the thermal hump has photon index $-1.8 \lesssim \alpha_{1} \lesssim-1.2$, whereas the distributed heated scenario yields a harder spectrum with -1.8 $\lesssim \alpha_{1} \lesssim 1$ for the chosen fiducial model parameters. The spectrum above the thermal hump has $\alpha_{2} \sim-1.8$ in both cases. While we do not carry out an exhaustive analysis to determine these two spectral slopes for different model parameters, and the cases shown here do not necessarily agree with the results of Ravasio et al. (2019), it remains to be seen if the two particle heating scenarios can find agreement with prompt emission spectra that show lowenergy breaks. The spectrum above the second hump shows distinct behaviour for the two particle heating cases. When the second hump forms due to Comptonization, the spectrum shows an exponential decline above the peak whereas in the fast-cooling synchrotron emission the spectrum declines more slowly. This feature can be potentially used to distinguish between the two scenarios by fitting to observations.

\section{SUMMARY AND DISCUSSION}

In this work, we have carried out self-consistent one-zone kinetic simulations of a photon-electron-positron plasma in a magnetized outflow with a striped-wind magnetic field structure. The flow is launched with high magnetization, $\sigma_{0} \gg 1$, which declines with radius as the flow expands and accelerates. The gradual dissipation of the magnetic field energy, either due to magnetic reconnection or MHD instabilities, accelerates the flow to terminal bulk Lorentz factors $\Gamma_{\infty}>100$. About half of the dissipated energy is assumed to heat the particles in the flow, out of which a fraction $\epsilon_{\mathrm{e}}$ is given to the electrons to power the prompt GRB emission. As the flow expands, the causal volume of the emission region expands with it. This brings in new particles into the causal region where the energy in the outflow is dissipated, a fraction $\xi \leq 1$ of which may be accelerated into a power-law energy distribution at magnetic reconnection sites. 

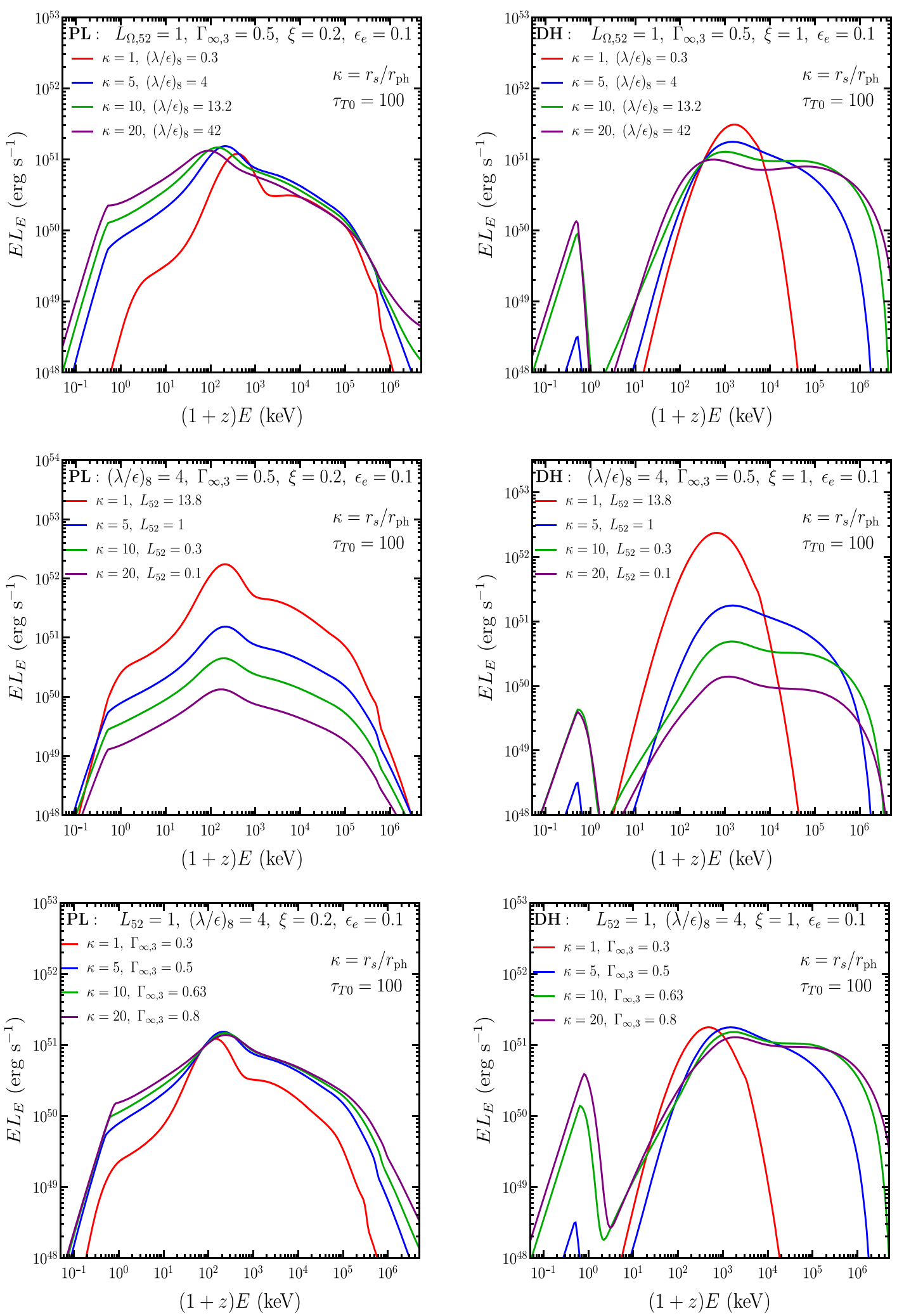

Figure 7. Spectra for the two heating scenarios, power-law particle injection (left) and distributed heating (right), shown for different values of $\kappa=r_{\mathrm{s}} / r_{\mathrm{ph}}$ and for correspondingly different values of $\left(\frac{\lambda}{\epsilon}\right)_{8}$ (top), $L_{52}$ (middle), and $\Gamma_{\infty, 3}$ (bottom) according to equation (28) while the remaining two parameters are kept fixed. 

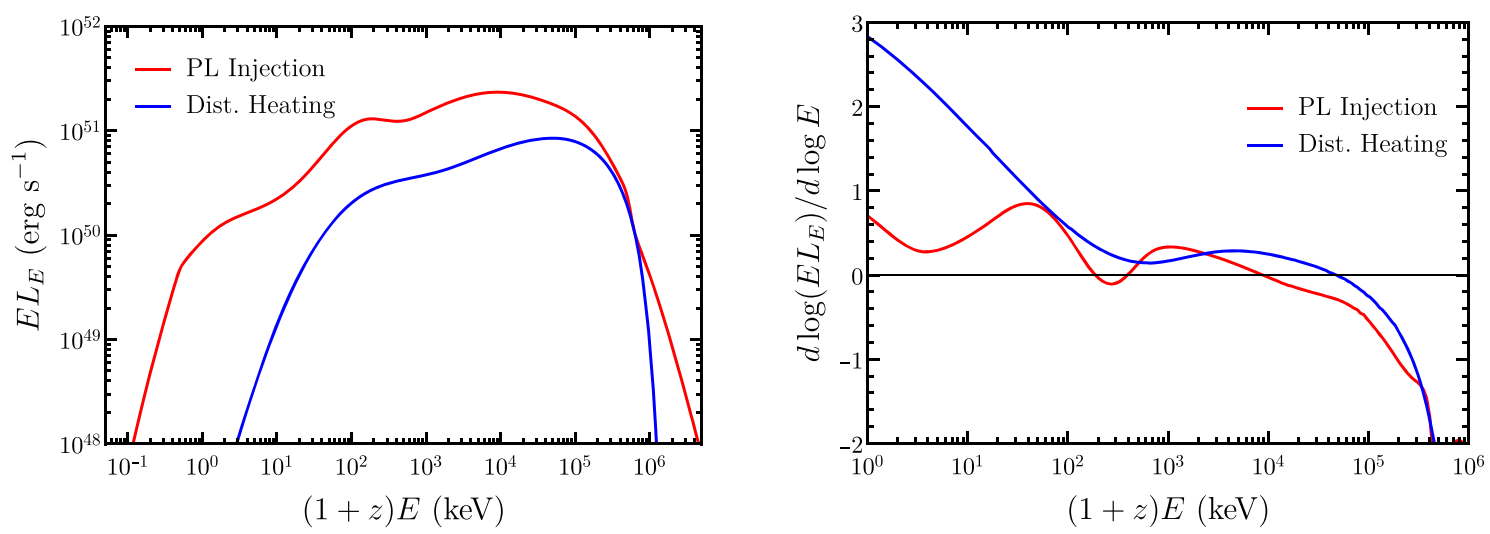

Figure 8. Left: Spectral break below the peak energy can be obtained in both particle heating scenarios. The model parameters for the two cases are (i) PL injection $-L_{52}=1, \Gamma_{\infty, 3}=0.5,\left(\frac{\lambda}{\epsilon}\right)_{8}=4, \epsilon_{\mathrm{e}}=0.4, \xi=0.2$, (ii) distributed heating $-L_{52}=0.1, \Gamma_{\infty, 3}=0.15,\left(\frac{\lambda}{\epsilon}\right)_{8}=10^{3}, \epsilon_{\mathrm{e}}=0.4, \xi=1$. Right: Spectral slopes for the two scenarios.

Alternatively, distributed heating by MHD instabilities in the flow may volumetrically heat all the particles $(\xi=1)$ at the same rate in the dissipation region. Gradual energy dissipation commences in the optically thick part of the flow $\left(r<r_{\mathrm{ph}}<r_{\mathrm{s}}\right)$ and the flow is continuously heated across the photospheric radius, as it becomes optically thin until $r=r_{\mathrm{s}}$. The underlying model has four to five parameters: (i) the jet power per unit solid angle $L_{\Omega}$, (ii) flow terminal LF $\Gamma_{\infty}$, (iii) characteristic length scale over which the magnetic field changes polarity in the lab-frame $\left(\frac{\lambda}{\epsilon}\right)$, (iv) efficiency of particle heating $\epsilon_{\mathrm{e}}$, and (v) fraction $\xi$ of injected electrons that are accelerated into a power-law energy distribution - not needed for the distributed heated scenario for which $\xi=1$.

The two particle heating scenarios lead to different spectra and corresponding particle distributions. In both cases, the spectrum exhibits two main components: a thermal component peaking at $(1+z) E_{\mathrm{th}} \sim 0.2-1 \mathrm{MeV}$ and a non-thermal component extending to high energies from the thermal peak. The origin of the non-thermal component is different in the two scenarios.

When power-law electrons are injected into the dissipation region, the non-thermal component arises due to the fast-cooling synchrotron emission. It dominates the spectrum below the thermal peak at energies $(1+z) E \lesssim 50 \mathrm{keV}$, where it becomes self-absorbed at $(1+$ $z) E \simeq 0.5 \mathrm{keV}$, and above the thermal peak at $1 \mathrm{MeV} \lesssim(1+z) E \lesssim$ $100 \mathrm{MeV}$, where the emission is suppressed at higher energies due to $\gamma \gamma$-annihilation. In our model, the spectral slope of the synchrotron emission at $E>E_{m}$, with spectral power $L_{E} \propto E^{-p / 2}$, depends on the power-law index $p=4 \sigma^{-0.3}$ of power-law electrons, which varies with the magnetization of the flow. This tends to produce high-energy spectra with photon indices $-2.5 \lesssim \beta \lesssim-2.2$, consistent with prompt GRB observations. Harder power-laws above the thermal peak that have been observed in only a few cases, e.g. GRB 090902B, can be produced for fixed values of electron power-law index with $p \sim$ 2 . The low-energy photon index, $-1.6 \lesssim \alpha \lesssim-1.2$ arises from the combination of the thermal component and the synchrotron emission at $E<E_{\mathrm{m}}$, for which $L_{E} \propto E^{-1 / 2}$.

When the dissipated energy is distributed among all the electrons (and the produced $e^{ \pm}$-pairs that are mostly sub-dominant in number in the cases simulated here), the non-thermal spectrum above the thermal peak arises due to Comptonization of the softer thermal peak photons. This also leads to softening of the spectrum below the thermal peak as the Compton- $y$ parameter grows above unity when the flow becomes optically thin. The thermal peak is pushed to higher energies at $(1+z) E \sim 1 \mathrm{MeV}$ and the photon index of the high-energy spectrum is $-2.6 \lesssim \beta \lesssim-2.0$ for $0.05 \lesssim \epsilon_{\mathrm{e}} \lesssim 0.2$, where the emission is suppressed at $(1+z) E \gtrsim 100 \mathrm{MeV}$ due to $\gamma \gamma$ annihilation. The low-energy photon index also depends on $\epsilon_{\mathrm{e}}$ with $\alpha$ $\gtrsim-1$ for $\epsilon_{\mathrm{e}} \lesssim 0.2$. A distinguishing feature of the distributed heating scenario is the appearance of soft X-ray emission at $(1+z) E \sim$ $0.5 \mathrm{keV}$ due to self-absorbed synchrotron emission from mildly relativistic mono-energetic particles.

Whether the final spectrum is Band-like or features a pre-dominant quasi-thermal component depends on $\kappa=r_{s} / r_{\mathrm{ph}}$, the ratio of the saturation radius to the photospheric radius, and which is always larger than unity in this work. For $\kappa \sim 1$, the final spectrum in both scenarios has a dominant quasi-thermal component since most of the energy is dissipated below the photosphere, which leads to suppression of the non-thermal component. On the other hand, when $\kappa>1$, the final spectrum is more Band-like where the nonthermal component build up as more energy is dissipated beyond the photosphere when the flow becomes optically thin. Since most GRBs do show a Band-like prompt spectrum, $\kappa$ is typically much larger than unity.

A broken power-law spectrum with spectral indices $\alpha_{1}=-2 / 3$ and $\alpha_{2}=-3 / 2$ below the $E L_{E}$-peak energy are expected from a fast cooling optically thin synchrotron emission for energies $E_{\mathrm{sa}}<E<$ $E_{c}<E_{m}$ and $E_{c}<E<E_{m}$, respectively (Sari et al. 1998). A serious argument against this emission model is the observation of harder low-energy spectral slopes in a sizable fraction of GRBs (Preece et al. 1998; Ghirlanda, Celotti \& Ghisellini 2003). Nevertheless, lowenergy spectral breaks at $E_{\mathrm{br}} \sim 50-100 \mathrm{keV}$ with spectral indices expected from optically thin synchrotron emission have been found in a number of GRBs (e.g. Oganesyan et al. 2017; Ravasio et al. 2018, 2019). If the break is indeed the cooling break, the energy of which scales with radius as $E_{c} \propto r^{3}$ (see equation 16), then having it at $\sim 100 \mathrm{keV}$ would demand that the emission must be at $r \gtrsim 3.4 \times 10^{15} \mathrm{~cm}$. At such a large radius the comoving magnetic field, with scaling $B^{\prime} \propto r^{-4 / 3}$, would reduce to merely $\lesssim 81 \mathrm{G}$. The constraints on the emission radius and the magnetic field become even more severe if dissipation occurs in a coasting flow (Beniamini \& Piran 2014; Ravasio et al. 2018, 2019). To circumvent these issues while keeping the same underlying emission model, Ghisellini et al. (2019) have recently proposed that the prompt GRB emission may be produced by power-law protons. However, knowing that protons are inefficient at radiating away their thermal 
energy as compared to electrons, the significant reduction in radiative efficiency must be compensated by having a much larger total energy budget, a requirement that may be too demanding. Alternatively, these conditions can be realized within magnetically dominated outflow models for the prompt emission, where dissipation is due to large-scale reconnection events that are triggered far from the central engine (Beniamini \& Granot 2016; Beniamini et al. 2018).

In this work, we show that low-energy breaks can be produced in the two particle heating scenarios considered here. This may avoid the need to invoke protons as the main radiators and to push the emission region farther out to large radii. At the same time, the main downside of the smaller emission radii required in this scenario explored here is that their associated variability time is significantly shorter than that observed in GRB light curves. The source of variability in the model explored here is therefore the variability of the engine itself. For the impulsive magnetic acceleration model $\lambda \sim c t_{v}$ and indeed $t_{v}$ should be reflected in the observed light-curve variability. Observationally, models invoking dissipation at relatively small emission radii (as considered here) and large emission radii (as discussed above) may be distinguished in the optical and $\gtrsim 100 \mathrm{MeV}$ bands. In both bands, models including dissipation at smaller radii should be strongly suppressed due to either synchrotron self-absorption (in the optical) or the pair-production threshold (above $\sim 100 \mathrm{MeV}$ ). Indeed, a significant fraction of GRBs show a suppression of $>100 \mathrm{MeV}$ flux that is consistent with the existence of a pair production threshold (Beniamini et al. 2011; Gill \& Granot 2018; Vianello et al. 2018).

The double-hump two-component spectrum has been seen in both the time-resolved and time-integrated spectra of a few short-hard (e.g. Guiriec et al. 2010) and long-soft GRBs (e.g. Guiriec et al. 2011). In all cases, it was shown that a Band function plus power law or a Band function plus blackbody spectrum resulted in a better fit over a single-component Band function. Furthermore, it was found that the non-thermal power-law component dominated the spectrum below few tens of $\mathrm{keV}$ and above $\sim \mathrm{MeV}$. This behaviour is the characteristic of having power-law electrons, as seen in the top left-hand panel of Fig. 2, where the non-thermal component is synchrotron emission. Double hump spectra were also produced in Gill \& Thompson (2014) who carried out one-zone kinetic simulations for a pure electronpositron plasma with strong magnetic fields. In that work, emission above the thermal peak was produced by inverse Compton scattering by mildly relativistic and mono-energetic $e^{ \pm}$-pairs; however, the flow was not continuously heated across the photosphere.

In two bright GRBs (080916C and 090926A) more than two spectral components have been shown to yield the best fit over a single Band function (Guiriec et al. 2015a). In this case, the first two components are the thermal and non-thermal, as discussed above, and the third component is modelled as a cut-off power law. In the models presented here, this third spectral component is difficult to obtain and might require a subdominant but hotter particle distribution in addition to the baryonic electrons. Since the mean energy per particle is assumed to be the same for all particles in the emission region, a separate hotter particle distribution cannot be obtained here.

The final spectrum from both particle heating scenarios shows good agreement with observations in general. To break the degeneracy between the two cases, additional diagnostics are needed. One such diagnostic is the suppression of X-ray emission for $(1+z) E \lesssim$ $1 \mathrm{keV}$ when power-law electrons are injected as compared to observable X-ray emission in the distributed heating case (also see e.g. Giannios 2008). Therefore, broad-band X-ray to gamma-ray observations (e.g. Page et al. 2007) during the prompt emission phase are needed to shed more light on this issue.
Another important diagnostic that can probe the underlying emission mechanism for the high-energy emission above the thermal peak is the detection of prompt linear polarization (e.g. Granot 2003; Gill, Granot \& Kumar 2020). Synchrotron emission is partially linearly polarized, and depending on the spectral index $s=-1$ $-\alpha$ when $L_{E} \propto E^{-s}$, the maximum polarization from an ordered magnetic field in the dissipation region is $0.5 \leq \Pi_{\max }=(s+1) /(s$ $+5 / 3) \lesssim 0.75$. Therefore, when synchrotron dominates the highenergy emission, as in the power-law electron injection case, high levels of polarization are expected. On the other hand, negligible polarization is expected when the high-energy emission is attributed to Comptonization. In this case, even though a singly Compton scattered photon is polarized, multiple such scatterings washes out the polarization when averaged over the entire GRB image on the plane of the sky. Polarization with $\Pi \lesssim 0.2$ is expected if the outflow has angular structure. Polarization is also expected from synchrotron emission below the thermal peak but above the self-absorption break in the scenario with power-law electron injection. Therefore, in this case, energy-resolved broad-band polarimetry should reveal a highlevel polarization at energies both below and above the peak but not near the peak.

Prompt GRB polarization in the range $0.1 \lesssim \Pi \lesssim 0.9$ has been possibly detected, albeit with only $\sim 3 \sigma$ significance in most cases, for a number of GRBs (see table 1 of Gill et al. 2020). However, a conclusive picture has not emerged yet. More sensitive upcoming/proposed X-ray and gamma-ray polarimetry missions, e.g. POLAR-II and eXTP, will be instrumental in furthering our understanding of the underlying prompt GRB emission mechanism.

\section{ACKNOWLEDGEMENTS}

RG thanks Sylvain Guiriec for useful discussions. RG and JG's research was supported by the ISF-NSFC joint research programme (grant no. 3296/19). PB's research was funded in part by the Gordon and Betty Moore Foundation through grant no. GBMF5076.

\section{DATA AVAILABILITY}

No new data were generated or analysed in support of this research.

\section{REFERENCES}

Abdo A. A. et al., 2009, ApJ, 706, L138

Abramowicz M. A., Novikov I. D., Paczynski B., 1991, ApJ, 369, 175

Ackermann M. et al., 2013, ApJ, 763, 71

Ackermann M. et al., 2014, Science, 343, 42

Band D. et al., 1993, ApJ, 413, 281

Bégué D., Pe'er A., 2015, ApJ, 802, 134

Bégué D., Pe'er A., Lyubarsky Y., 2017, MNRAS, 467, 2594

Beloborodov A. M., 2010, MNRAS, 407, 1033

Beloborodov A. M., 2011, ApJ, 737, 68

Beloborodov A. M., 2013, ApJ, 764, 157

Beloborodov A. M., Mészáros P., 2017, Space Sci. Rev., 207, 87

Beniamini P., Giannios D., 2017, MNRAS, 468, 3202

Beniamini P., Granot J., 2016, MNRAS, 459, 3635

Beniamini P., Piran T., 2013, ApJ, 769, 69

Beniamini P., Piran T., 2014, MNRAS, 445, 3892

Beniamini P., Guetta D., Nakar E., Piran T., 2011, MNRAS, 416, 3089

Beniamini P., Barniol Duran R., Giannios D., 2018, MNRAS, 476, 1785

Bhattacharya M., Kumar P., 2020, MNRAS, 491, 4656

Briggs M. S. et al., 1999, ApJ, 524, 82

Daigne F., Bošnjak Ž., Dubus G., 2011, A\&A, 526, A110

Drenkhahn G., 2002, A\&A, 387, 714

Drenkhahn G., Spruit H. C., 2002, A\&A, 391, 1141 
Eichler D., Levinson A., 2000, ApJ, 529, 146

Ghirlanda G., Celotti A., Ghisellini G., 2003, A\&A, 406, 879

Ghisellini G. et al., 2019, A\&A, 636A, 82

Ghisellini G., Celotti A., 1999, ApJ, 511, L93

Giannios D., 2006, A\&A, 457, 763

Giannios D., 2008, A\&A, 480, 305

Giannios D., Spruit H. C., 2005, A\&A, 430, 1

Giannios D., Spruit H. C., 2007, A\&A, 469, 1

Gill R., Granot J., 2018, MNRAS, 475, L1

Gill R., Thompson C., 2014, ApJ, 796, 81

Gill R., Granot J., Lyubarsky Y., 2018, MNRAS, 474, 3535

Gill R., Granot J., Kumar P., 2020, MNRAS, 491, 3343

Granot J., 2003, ApJ, 596, L17

Granot J., 2012, MNRAS, 421, 2467

Granot J., Sari R., 2002, ApJ, 568, 820

Granot J., Piran T., Sari R., 1999, ApJ, 513, 679

Granot J., Piran T., Sari R., 2000, ApJ, 534, L163

Granot J., Cohen-Tanugi J., Silva E. D. C. E., 2008, ApJ, 677, 92

Granot J., Komissarov S. S., Spitkovsky A., 2011, MNRAS, 411, 1323

Guiriec S. et al., 2010, ApJ, 725, 225

Guiriec S. et al., 2011, ApJ, 727, L33

Guiriec S. et al., 2015a, ApJ, 807, 148

Guiriec S. et al., 2016b, ApJ, 831, L8

Guiriec S., Mochkovitch R., Piran T., Daigne F., Kouveliotou C., Racusin J., Gehrels N., McEnery J., 2015b, ApJ, 814, 10

Guiriec S., Gonzalez M. M., Sacahui J. R., Kouveliotou C., Gehrels N., McEnery J., 2016a, ApJ, 819, 79

Guiriec S., Gehrels N., McEnery J., Kouveliotou C., Hartmann D. H., 2017, ApJ, 846, 138

Guo F., Liu Y.-H., Daughton W., Li H., 2015, ApJ, 806, 167

Kagan D., Sironi L., Cerutti B., Giannios D., 2015, Space Sci. Rev., 191, 545

Kaneko Y., Preece R. D., Briggs M. S., Paciesas W. S., Meegan C. A., Band D. L., 2006, ApJS, 166, 298

Katz J. I., 1994, ApJ, 432, L107

Komissarov S. S., 2012, MNRAS, 422, 326

Kouveliotou C., Meegan C. A., Fishman G. J., Bhat N. P., Briggs M. S., Koshut T. M., Paciesas W. S., Pendleton G. N., 1993, ApJ, 413, L101

Kumar P., McMahon E., 2008, MNRAS, 384, 33

Kumar P., Zhang B., 2015, Phys. Rep., 561, 1

Lightman A. P., Zdziarski A. A., 1987, ApJ, 319, 643

Lyubarsky Y., 2010, ApJ, 725, L234

Lyubarsky Y., Kirk J. G., 2001, ApJ, 547, 437

Lyutikov M., Blandford R., 2003, preprint (ArXiv:astro-ph/0312347)

McKinney J. C., Uzdensky D. A., 2012, MNRAS, 419, 573

Mészáros P., Rees M. J., 2000, ApJ, 530, 292

Metzger B. D., Giannios D., Thompson T. A., Bucciantini N., Quataert E., 2011, MNRAS, 413, 2031

Oganesyan G., Nava L., Ghirlanda G., Celotti A., 2017, ApJ, 846, 137

Page K. L. et al., 2007, ApJ, 663, 1125

Parfrey K., Giannios D., Beloborodov A. M., 2015, MNRAS, 446, L61

Pe'er A., 2008, ApJ, 682, 463

Pe'er A., 2017, ApJ, 850, 200

Pe'er A., Waxman E., 2004, ApJ, 613, 448

Pe'er A., Waxman E., 2005, ApJ, 628, 857

Pe’er A., Mészáros P., Rees M. J., 2006, ApJ, 642, 995

Piran T., 2004, Rev. Mod. Phys., 76, 1143

Preece R. D., Briggs M. S., Mallozzi R. S., Pendleton G. N., Paciesas W. S., Band D. L., 1998, ApJ, 506, L23

Preece R. D., Briggs M. S., Mallozzi R. S., Pendleton G. N., Paciesas W. S., Band D. L., 2000, ApJS, 126, 19

Ravasio M. E., Oganesyan G., Ghirlanda G., Nava L., Ghisellini G., Pescalli A., Celotti A., 2018, A\&A, 613, A16

Ravasio M. E., Ghirlanda G., Nava L., Ghisellini G., 2019, A\&A, 625, A60

Rees M. J., Meszaros P., 1994, ApJ, 430, L93

Rees M. J., Mészáros P., 2005, ApJ, 628, 847

Ryde F., 2004, ApJ, 614, 827

Ryde F., 2005, ApJ, 625, L95

Sari R., Piran T., Narayan R., 1998, ApJ, 497, L17
Sironi L., Spitkovsky A., 2014, ApJ, 783, L21

Stern B. E., Poutanen J., 2004, MNRAS, 352, L35

Tavani M., 1996, ApJ, 466, 768

Thompson C., 1994, MNRAS, 270, 480

Thompson C., Gill R., 2014, ApJ, 791, 46

Vianello G., Gill R., Granot J., Omodei N., Cohen-Tanugi J., Longo F., 2018, ApJ, 864, 163

Vurm I., Beloborodov A. M., 2016, ApJ, 831, 175

Vurm I., Beloborodov A. M., Poutanen J., 2011, ApJ, 738, 77

Vurm I., Lyubarsky Y., Piran T., 2013, ApJ, 764, 143

Werner G. R., Uzdensky D. A., Cerutti B., Nalewajko K., Begelman M. C., 2016, ApJ, 816, L8

\section{APPENDIX: PARTICLE INJECTION AND HEATING}

We briefly describe here how the two particle heating scenarios are implemented in this work.

The comoving number density of baryonic electrons in the flow at any given radius $r$ can be expressed as

$n^{\prime}(r)=\frac{L_{\Omega}}{\Gamma_{\infty} m_{\mathrm{p}} c^{3}} \frac{1}{r^{2} \Gamma} \propto r^{-7 / 3}$,

where the expression after the proportionality assumes $r<r_{\mathrm{s}}$ for which $\Gamma \propto r^{1 / 3}$. The number density of particles in the comoving causal volume $\tilde{V}^{\prime}=4 \pi r^{3} / \Gamma$ is the same as above, such that $\tilde{n}^{\prime}(r)=$ $n^{\prime}(r)$. The number of particles in the causal volume $\tilde{N}(r)$ grows with radius as the volume expands,

$\tilde{N}(r)=n^{\prime}(r) \tilde{V}^{\prime}=\tilde{n}^{\prime}(r) \tilde{V}^{\prime}=\frac{4 \pi L_{\Omega}}{\Gamma_{\infty} m_{\mathrm{p}} c^{3}} \frac{r}{\Gamma^{2}} \propto r^{1 / 3}$,

where this represents a fraction $\left(r / r_{\mathrm{s}}\right)^{1 / 3}$ of the total particle number $N$ in the lab-frame volume of a spherical shell whose characteristic width $\lambda \approx r_{s} / \Gamma_{\infty}^{2}$ remains constant. Since no external particles are added to this shell as it expands, $N$ remains constant. Therefore, when $r=r_{\mathrm{s}}$ all the particles in this shell are found within the causal volume.

The one-zone kinetic code used in this work (see Gill \& Thompson 2014 for more details) evolves particle number density instead of particle number in the causal volume. The evolution of electron number density distribution as energy is added in the form of powerlaw particles can be obtained from the evolution of the particle number distribution

$\frac{\partial \tilde{N}\left(p_{\mathrm{e}}\right)}{\partial t^{\prime}}=\frac{\partial}{\partial p_{\mathrm{e}}}\left[\frac{\gamma_{\mathrm{e}}}{p_{\mathrm{e}}} A_{\mathrm{ad}} \tilde{N}\left(p_{\mathrm{e}}\right)\right]+S^{\prime}\left(p_{\mathrm{e}}\right)$,

where $\tilde{N}\left(p_{\mathrm{e}}\right)=\partial \tilde{N} / \partial p_{\mathrm{e}}$ is the momentum space particle number distribution. The first term on the RHS describes the movement of particles in momentum space, using a Fokker-Planck advection term, due to adiabatic cooling $\left(A_{\mathrm{ad}}\right)$, and in general other energy exchange processes, namely Compton scattering, synchrotron cooling, and Coulomb cooling, that are not discussed here but included in the numerical code. The second term is the source term, $S^{\prime}\left(p_{\mathrm{e}}\right)=$ $\mathrm{d} \tilde{N} / \mathrm{d} t^{\prime} \mathrm{d} p_{\mathrm{e}}$, which describes the injection of new power-law particles into the causal volume. In general, $S\left(p_{\mathrm{e}}\right)$ receives contributions from other processes, such as Compton scattering, pair-production and annihilation.

The advection coefficient for the adiabatic cooling of particles is given by

$A_{\mathrm{ad}}=-\frac{1}{m_{\mathrm{e}} c^{2}} \frac{\mathrm{d} E_{\mathrm{e}}^{\prime}}{\mathrm{d} t^{\prime}}=\frac{(\hat{\gamma}-1) E_{\mathrm{e}}^{\prime}}{m_{\mathrm{e}} c^{2}} \frac{\mathrm{d} \ln V^{\prime}}{\mathrm{d} t^{\prime}}=\frac{(\hat{\gamma}-1)\left(\gamma_{\mathrm{e}}-1\right)}{t_{\mathrm{ad}}^{\prime}}$. 
Here, we have used the scaling of particle energy with comoving volume due to adiabatic expansion, such that $E_{\mathrm{e}}^{\prime}=\left(\gamma_{\mathrm{e}}-1\right) m_{\mathrm{e}} c^{2} \propto$ $V^{\prime 1-\hat{\gamma}}$ where $\hat{\gamma}=4 / 3(5 / 3)$ is the adiabatic index for a relativistic (non-relativistic) particle distribution. In general, the advection coefficient includes contributions from other processes that change the energy of particles, e.g. Compton scattering, synchrotron cooling, Coulomb interactions, and particle heating (as described below). The adiabatic cooling timescale is obtained from the rate of volume expansion, where

$\frac{1}{t_{\mathrm{ad}}^{\prime}}=\frac{\mathrm{d} \ln V^{\prime}}{\mathrm{d} t^{\prime}}=\frac{1}{r^{2} \Gamma} \frac{\mathrm{d}\left(r^{2} \Gamma\right)}{\mathrm{d} t^{\prime}}=\frac{c}{r^{2}} \frac{\mathrm{d}\left(r^{2} \Gamma\right)}{\mathrm{d} r}=\zeta \frac{\Gamma c}{r}$,

where $\zeta=7 / 3$ for $r<r_{\mathrm{s}}$ when $\Gamma \propto r^{1 / 3}$, and $\zeta=2$ for $r \geq r_{\mathrm{s}}$ when $\Gamma=\Gamma_{\infty}$.

By expressing the LHS of equation (A3) using the number density, we find

$\frac{\partial \tilde{n}^{\prime}\left(p_{\mathrm{e}}\right)}{\partial t^{\prime}}=\frac{\partial}{\partial t^{\prime}}\left(\frac{\tilde{N}\left(p_{\mathrm{e}}\right)}{\tilde{V}^{\prime}}\right)=\frac{1}{\tilde{V}^{\prime}} \frac{\partial \tilde{N}\left(p_{\mathrm{e}}\right)}{\partial t^{\prime}}-\frac{\tilde{n}^{\prime}\left(p_{\mathrm{e}}\right)}{t_{\tilde{V}^{\prime}}^{\prime}}$,

where we have conveniently defined the comoving growth time of the causal volume, $t_{\tilde{V}^{\prime}}^{\prime}$, through

$\frac{1}{t_{\tilde{V}^{\prime}}^{\prime}}=\frac{\mathrm{d} \ln \tilde{V}^{\prime}}{\mathrm{d} t^{\prime}}$

Next, we use equation (A2) to express the rate of change of the comoving number density with comoving time in terms of $\tilde{N}$ and the causal volume $\tilde{V}^{\prime}$, so that

$\frac{\mathrm{d} \ln \tilde{n}^{\prime}}{\mathrm{d} t^{\prime}}=\frac{\mathrm{d} \ln n^{\prime}}{\mathrm{d} t^{\prime}}=-\frac{\mathrm{d} \ln V^{\prime}}{\mathrm{d} t^{\prime}}=\frac{\mathrm{d} \ln \tilde{N}}{\mathrm{~d} t^{\prime}}-\frac{\mathrm{d} \ln \tilde{V}^{\prime}}{\mathrm{d} t^{\prime}}=-\frac{1}{t_{\mathrm{ad}}^{\prime}}=\frac{\dot{\tilde{n}}^{\prime}}{\tilde{n}^{\prime}}$,

which in turn implies

$$
\frac{1}{t_{\tilde{V}^{\prime}}^{\prime}}=\frac{1}{t_{\mathrm{ad}}^{\prime}}+\frac{1}{t_{\mathrm{inj}}^{\prime}}
$$

where the comoving particle injection time into the causal volume is defined by

$$
\frac{1}{t_{\text {inj }}^{\prime}}=\frac{\mathrm{d} \ln \tilde{N}}{\mathrm{~d} t^{\prime}}=\frac{\mathrm{d} \ln \tilde{V}^{\prime}}{\mathrm{d} t^{\prime}}+\frac{\mathrm{d} \ln \tilde{n}^{\prime}}{\mathrm{d} t^{\prime}}=\frac{1}{t_{\tilde{V}^{\prime}}^{\prime}}+\frac{\dot{\tilde{n}}^{\prime}}{\tilde{n}^{\prime}}=\frac{1}{t_{\tilde{V}^{\prime}}^{\prime}}-\frac{1}{t_{\mathrm{ad}}^{\prime}},
$$

and $\dot{n}_{\text {inj }}^{\prime}$ is the particle injection rate per unit volume. Note that the causal comoving volume $\tilde{V}^{\prime}$ grows faster than the total comoving volume $V^{\prime}$ since it occupies an increasing fraction of it, $f=\tilde{V}^{\prime} / V^{\prime}=$ $\tilde{N} / N=\min \left[1,\left(r / r_{s}\right)^{1 / 3}\right]$, where $1 / t_{\text {inj }}^{\prime}=d \ln f / d t^{\prime}$.

Now, using equations (A3) and (A6) lead to the equation for the number density evolution

$$
\begin{aligned}
\frac{\partial \tilde{n}^{\prime}\left(p_{\mathrm{e}}\right)}{\partial t^{\prime}} & =\frac{\partial}{\partial p_{\mathrm{e}}}\left[\frac{\gamma_{\mathrm{e}}}{p_{\mathrm{e}}} A_{\mathrm{ad}} \frac{\tilde{N}\left(p_{\mathrm{e}}\right)}{\tilde{V}^{\prime}}\right]+\frac{S^{\prime}\left(p_{\mathrm{e}}\right)}{\tilde{V}^{\prime}}-\frac{\tilde{n}^{\prime}\left(p_{\mathrm{e}}\right)}{t_{\tilde{V}^{\prime}}^{\prime}} \\
& =\frac{\partial}{\partial p_{\mathrm{e}}}\left[\frac{\gamma_{\mathrm{e}}}{p_{\mathrm{e}}} A_{\mathrm{ad}} n^{\prime}\left(p_{\mathrm{e}}\right)\right]+Q^{\prime}\left(p_{\mathrm{e}}\right)-\frac{\tilde{n}^{\prime}\left(p_{\mathrm{e}}\right)}{t_{\tilde{V}^{\prime}}^{\prime}},
\end{aligned}
$$

where $Q^{\prime}\left(p_{\mathrm{e}}\right)=S^{\prime}\left(p_{\mathrm{e}}\right) / \tilde{V}^{\prime}=\mathrm{d} \tilde{N} / \mathrm{d} \tilde{V}^{\prime} \mathrm{d} t^{\prime} \mathrm{d} p_{e}$. By integrating the above equation over $p_{\mathrm{e}}$, it can be shown that the adiabatic cooling term vanishes and $Q^{\prime}=\int Q^{\prime}\left(p_{\mathrm{e}}\right) \mathrm{d} p_{\mathrm{e}}=S^{\prime} / \tilde{V}^{\prime}=\tilde{n}^{\prime} / t_{\text {inj }}^{\prime}$, where $S^{\prime}=\int S^{\prime}\left(p_{\mathrm{e}}\right) \mathrm{d} p_{e}=\mathrm{d} \tilde{N} / \mathrm{d} t^{\prime}$ so that the equation reads $\mathrm{d} \tilde{n}^{\prime} / \mathrm{d} t^{\prime}=$ $\left(1 / t_{\mathrm{inj}}^{\prime}-1 / t_{\tilde{V}^{\prime}}^{\prime}\right) \tilde{n}^{\prime}=-\tilde{n}^{\prime} / t_{\mathrm{ad}}^{\prime}=\tilde{n}^{\prime}\left(\mathrm{d} \ln \tilde{n}^{\prime} / \mathrm{d} t^{\prime}\right)=\dot{\tilde{n}}^{\prime}$.

The comoving rate of particle injection per unit volume per unit dimensionless momentum comprises of two terms, where a fraction $\xi$ of the total is accelerated into a power-law (non-thermal) distribution and the remaining fraction $(1-\xi)$ forms a thermal distribution,

$$
\begin{aligned}
Q^{\prime}\left(p_{e}\right) & =(1-\xi) Q^{\prime}\left(p_{e}\right)+\xi Q^{\prime}\left(p_{e}\right) \\
& =(1-\xi) Q^{\prime} \hat{Q}_{\mathrm{th}}^{\prime}\left(p_{e}\right)+\xi Q^{\prime} \hat{Q}_{\mathrm{nth}}^{\prime}\left(p_{e}\right),
\end{aligned}
$$

where $\hat{Q}^{\prime}\left(p_{e}\right)=Q^{\prime}\left(p_{e}\right) / Q^{\prime}$ represents the normalized momentum distribution. The total energy density per unit rest mass energy of the injected distribution can be expressed as

$$
\int\left(\gamma_{\mathrm{e}}-1\right) Q^{\prime}\left(p_{\mathrm{e}}\right) \mathrm{d} p=Q^{\prime}\left[(1-\xi)\left(\left\langle\gamma_{\mathrm{e}}\right\rangle_{\mathrm{th}}-1\right)+\xi\left(\left\langle\gamma_{\mathrm{e}}\right\rangle_{\mathrm{nth}}-1\right)\right]
$$

where $\left\langle\gamma_{e}\right\rangle_{\text {th }}$ is the mean energy per rest mass energy of the thermal distribution and for the power-law distribution $\left\langle\gamma_{e}\right\rangle_{\text {nth }}$ is given in equation (10). Here, we make the assumption that the injected thermal particles are cold, so that $\left\langle\gamma_{e}\right\rangle_{\text {th }}-1 \ll \frac{\xi}{1-\xi}\left(\left\langle\gamma_{e}\right\rangle_{\text {nth }}-1\right)$. The momentum distribution of power-law electrons is given by

$Q_{\mathrm{nth}}^{\prime}\left(p_{\mathrm{e}}\right)=\frac{d \gamma_{\mathrm{e}}}{d p_{\mathrm{e}}} Q_{\mathrm{nth}}^{\prime}\left(\gamma_{\mathrm{e}}\right)=\frac{p_{\mathrm{e}}}{\gamma_{\mathrm{e}}} Q_{\mathrm{nth}}^{\prime}\left(\gamma_{\mathrm{e}}\right)=Q_{0}^{\prime} p_{\mathrm{e}} \gamma_{\mathrm{e}}^{-p-1}$.

The normalization $Q_{0}^{\prime}$ is obtained by equating the rate of energy injection per unit volume to that given to the electrons due to dissipation, such that

$$
\frac{1}{m_{\mathrm{e}} c^{2}} \frac{\mathrm{d} U_{\mathrm{e}}^{\prime}}{\mathrm{d} t^{\prime}}=\int\left(\gamma_{\mathrm{e}}-1\right) Q_{\mathrm{nth}}^{\prime}\left(p_{\mathrm{e}}\right) \mathrm{d} p_{\mathrm{e}}=\int\left(\gamma_{\mathrm{e}}-1\right) Q_{\mathrm{nth}}^{\prime}\left(\gamma_{\mathrm{e}}\right) \mathrm{d} \gamma_{\mathrm{e}},
$$

which for $\gamma_{M}>\gamma_{m} \gg 1$ gives

$$
Q_{0}^{\prime}=\frac{(p-2)}{\left(\gamma_{m}^{2-p}-\gamma_{M}^{2-p}\right)} \frac{\epsilon_{\mathrm{e}}}{2 m_{\mathrm{e}} c^{2}} \frac{\mathrm{d} U_{\mathrm{diss}}^{\prime}}{\mathrm{d} t^{\prime}}
$$

where we take $\gamma_{M}=\gamma_{\max }$, the maximum LF corresponding to the outer boundary of the particle momentum grid in the simulation. The efficiency of acceleration is controlled by the mean energy of the power-law electrons, which depends on $\xi$, and ultimately by the minimal energy of power-law electrons $\gamma_{m}=[(p-2) /(p-$ 1)] $\left\langle\gamma_{\mathrm{e}}\right\rangle_{\mathrm{nth}}$.

In the distributed heating scenario, all particles (including produced $e^{ \pm}$-pairs) in the causal volume are heated at the same rate. This volumetric heating can again be described using a FokkerPlanck equation with only the advective term

$$
\frac{\partial \tilde{n}_{ \pm}^{\prime}\left(p_{\mathrm{e}}\right)}{\partial t^{\prime}}=\frac{\partial}{\partial p_{\mathrm{e}}}\left[\frac{\gamma_{\mathrm{e}}}{p_{\mathrm{e}}}\left(A_{\mathrm{ad}}+A_{ \pm, \text {heat }}\right) \tilde{n}_{ \pm}^{\prime}\left(p_{\mathrm{e}}\right)\right]-\frac{\tilde{n}_{ \pm}^{\prime}\left(p_{\mathrm{e}}\right)}{t_{\mathrm{ad}}^{\prime}},
$$

where $A_{ \pm \text {,heat }}$ is the advection coefficient. The rate of change of particle energy due to heating is obtained from the volumetric rate of energy injection that yields

$A_{ \pm, \text {heat }}=\mathcal{A} \frac{\tilde{n}_{ \pm}^{\prime}}{\left(\tilde{n}_{+}^{\prime}+\tilde{n}_{-}^{\prime}\right)} \frac{\epsilon_{\mathrm{e}}}{2 m_{\mathrm{e}} c^{2}} \frac{\mathrm{d} U_{\mathrm{diss}}^{\prime}}{\mathrm{d} t^{\prime}}$,

where the normalization is given by

$\mathcal{A}=\left[\left\{\frac{\gamma_{\mathrm{e}}^{2}}{p_{\mathrm{e}}} \tilde{n}_{ \pm}^{\prime}\left(p_{\mathrm{e}}\right)\right\}_{p_{\mathrm{e}, \min }}^{p_{\mathrm{e}, \max }}-\tilde{n}_{ \pm}^{\prime}\right]^{-1}$

with the term in braces evaluated at the particle momentum grid boundaries. The electron and positron distributions are evolved separately as described by the above equations with '-' and ' + ' subscripts, respectively.

This paper has been typeset from a $\mathrm{T}_{\mathrm{E}} \mathrm{X} / \mathrm{L} \mathrm{T} \mathrm{E} \mathrm{X}$ file prepared by the author. 